\title{
Protection cathodique appliquée au béton armé aérien : étude expérimentale et modélisation
}

\author{
Raoul François* \\ LMDC, INSA, UPS, Université de Toulouse, Toulouse, France
}

Reçu le 12 décembre 2017 / Accepté le 11 avril 2018

\begin{abstract}
Résumé. Cet article présente une étude expérimentale et numérique de la protection cathodique par courant imposé appliquée sur un mur en béton armé soumis à une corrosion localisée (par macro-piles), typique du béton armé. L'étude numérique est réalisée avec le logiciel COMSOL Multiphysics. L'étude expérimentale et l'étude numérique basée sur les lois physiques de base qui régissent les phénomènes électrochimiques confirment que la polarisation des systèmes de corrosion de type macro-piles est hétérogène et ne peut être appréhendée qu'en trois dimensions. L'uniformité du champ de potentiel à l'interface acier-béton n'est physiquement pas réalisable en appliquant un courant de protection de la surface du béton. Le principe général de la protection cathodique n'a pas à être discuté, mais une attention particulière doit être accordée à la réponse non uniforme des systèmes de corrosion par macro-piles car il remet en question, entre autres, le choix de la position des témoins où la performance du système de PC est évaluée et l'utilisation de critères exprimés en potentiels.
\end{abstract}

Mots clés : béton armé / corrosion localisée / chlorures / protection cathodique par courant imposé / critères d'efficacité / critères de fonctionnement / polarisation / dépolarisation

\begin{abstract}
Cathodic protection applied to reinforced concrete structures: experimental study and modelling. This article presents an experimental and numerical study of the impressed current cathodic protection applied on a reinforced concrete wall subjected to localized corrosion (macro-cell corrosion), typical of the reinforced concrete. The numerical study is carried out with COMSOL Multiphysics software. Experimental and numerical studies based on the basic physical laws that govern the electrochemical phenomena, confirm that the polarization of macro-cell corrosion systems is heterogeneous and can only be modelled in three dimensions. The uniformity of the potential field at the steel-concrete interface is physically not achievable by applying a protective current of the concrete surface. The general principle of cathodic protection does not have to be discussed, but special attention must be paid to the non-uniform response of macro-cell corrosion systems because it calls into question, among other things, the choice of the location of embedded probes where the performance of the PC system is evaluated and the use of criteria expressed in potentials.
\end{abstract}

Keywords: reinforced concrete / localized corrosion / chlorides / impressed current cathodic protection / performance criteria / polarization / depolarization

\section{Préambule}

Cet article est extrait du mémoire présenté pour obtenir une certification (CEFRACOR Certification) en protection cathodique secteur Béton de niveau 3 par rapport à la norme EN 15257 en 2016. Ce niveau de certification est équivalent depuis le $1^{\mathrm{er}}$ janvier 2018 au niveau 4 par rapport à la norme EN ISO 15257:2017.

e-mail: raoul.francois@insa-toulouse.fr

\section{Introduction}

Cet article est consacré à une étude expérimentale et numérique d'un système de protection cathodique des armatures du béton armé par courant imposé. Contrairement à la corrosion atmosphérique et à la corrosion en solution, la corrosion des aciers d'armature dans le béton est non uniforme, c'est-à-dire avec une séparation spatiale des zones anodiques et cathodiques: on utilise aussi les appellations corrosion localisée ou corrosion par macropiles (macro-cell en anglais). La corrosion des armatures dans le béton armé est différente de la corrosion atmosphérique en raison de la nature de l'électrolyte: le 


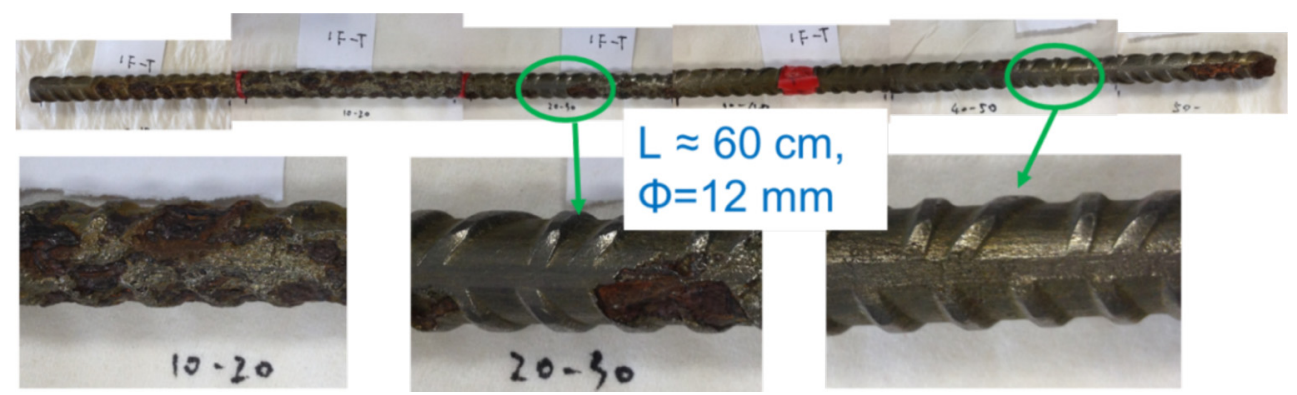

Fig. 1. État de corrosion non uniforme d'une armature longitudinale extraite d'une poutre en béton armé exposée 27 mois à une corrosion naturelle en présence de cycles de mouillage-séchage d'un brouillard salin : début des expérimentations en 2010 d'après Yu et al. [6].

béton, mais aussi parce que les armatures ne sont pas dans un état «homogène». Le béton possède une résistivité élevée (qui dépend entre autres du degré de saturation) et un pH élevé $(>12,5)$. Le pH élevé conduit à une protection des armatures en acier vis-à-vis de la corrosion: ce phénomène appelé passivité correspond en fait à une vitesse de corrosion très faible. La résistivité du béton élevée conduit à une non-uniformisation des potentiels des aciers lorsque ceux-ci sont modifiés par la présence des deux phénomènes pouvant dépassiver l'acier: la présence de chlorures ou la baisse de $\mathrm{pH}$ due à la carbonatation. En plus des aspects résistivité élevée et pH élevé, d'autres phénomènes conduisent à une corrosion non uniforme: la non-homogénéité de la pénétration des chlorures et du phénomène de carbonatation (zones en état de traction, pénétration préférentielle aux interfaces pâtes-granulats [1], la non-homogénéité de l'interface acier-béton due en particulier au phénomène de ressuage, la présence de plusieurs lits d'armatures qui conduit à la coexistence d'armatures à l'état passif et d'armatures présentant des zones actives de corrosion pendant la propagation de la corrosion.

Le développement de la corrosion due à la présence de chlorures est différent de celui lié à la carbonatation : aussi, il est préférable de séparer les deux processus. Ainsi, cet article sera plus spécifiquement consacré à l'étude de la corrosion en présence de chlorures et son contrôle par une protection cathodique. Le processus de la corrosion en présence de chlorures est étudié par de nombreux chercheurs et ingénieurs civils depuis plus d'un demisiècle, sans déboucher sur un consensus. Le manque de compréhension fondamental est également mis en évidence par le fait que les politiques relatives aux structures corrodées diffèrent sensiblement d'un pays à l'autre (malgré la similitude du problème technique) [2], et que les stratégies d'ingénierie actuelles reposent en grande partie sur l'expérience passée et sont empiriques [2,3]. L'approche conceptuelle de l'initiation de la corrosion est basée sur la définition d'une valeur de seuil de chlorure $\left(C_{c r i t}\right)$ qui conduirait à une dépassivation de l'acier. Malgré de nombreuses tentatives de recherche pour déterminer $C_{c r i t}$ aucun accord n'a toutefois été conclu sur une valeur [4]. En fait, la dispersion de la littérature extrêmement riche au sujet du $C_{\text {crit }}$ suggère que d'autres paramètres (autres que la concentration en chlorure) jouent un rôle important et qu'ils ne sont pas encore bien compris et pris en compte dans le concept actuel. Il n'y a pas de doute que la concentration de chlorure joue un rôle important et c'est un paramètre qui peut être facilement mesuré. Néanmoins, il n'y a aujourd'hui aucune confirmation que c'est le paramètre le plus important dans l'initiation de corrosion. L'introduction d'autres concepts comme les «défauts »de l'interface acier-béton [5] font partie des palliatifs à l'absence de compréhension et de signification du concept de $C_{\text {crit }}$.

Dans le béton armé, le processus de corrosion des aciers d'armature résulte majoritairement de la formation de macro-piles. Ainsi la figure 1 montre l'état de corrosion d'une armature longitudinale extraite d'une poutre en béton armé soumise à une corrosion naturelle dans un environnement constitué de cycles de mouillage-séchage avec un brouillard salin. Le long de l'armature, on observe des zones de corrosion (anodique) et des zones non corrodées (cathodique).

De plus sur une même section de barre, la corrosion n'est pas non plus uniforme le long du périmètre (Fig. 2). Les raisons peuvent être reliées à la qualité de l'interface acier-béton qui est affectée par la présence des armatures pendant la prise du béton: phénomène de tassement et de ressuage du béton frais [7-9].

Même la corrosion à long terme reste principalement localisée et ce en dépit de conditions environnementales devant a priori conduire à une corrosion généralisée. Attention cependant, ce point n'est vrai qu'en l'absence de fissures induites par la corrosion : en effet, ces dernières qui sont parallèles aux armatures conduisent à une généralisation de la corrosion se superposant à la corrosion localisée [10]. La figure 3 montre un cadre d'effort tranchant extrait d'une poutre corrodée naturellement 28 ans dans un environnement salin. Le taux de chlorures totaux mesuré en tout point du cadre est supérieur à $2 \%$ par rapport à la masse de ciment et conduirait à la conclusion d'une initiation de la corrosion sur toute la longueur d'armature par rapport au concept de seuil critique en chlorures $C_{c r i t}$ (en prenant la valeur usuelle de 0,4\% considérée comme seuil d'amorçage de la corrosion). Cependant, seule une petite partie du cadre d'effort tranchant est corrodée, la majeure partie est restée non corrodée et assure le rôle de cathode dans le processus de corrosion localisée.

Une méthode fiable pour quantifier la vitesse de corrosion dans le béton serait essentielle pour évaluer la durée de vie résiduelle des structures en béton armé. En 


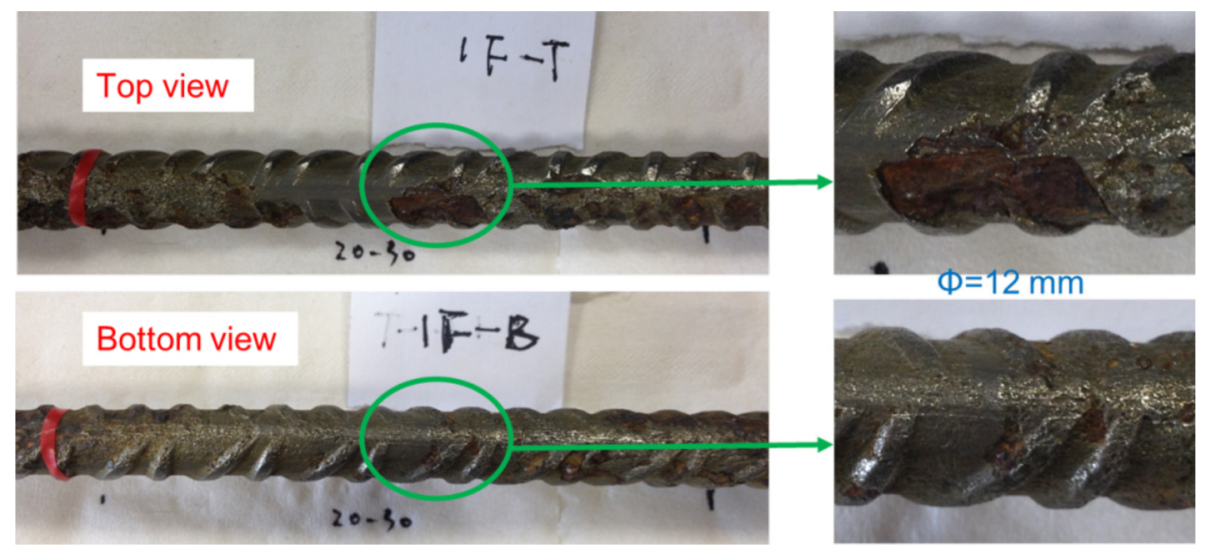

Fig. 2. Non-uniformité de la corrosion le long du périmètre d'une barre d'armature en acier extraite d'une poutre en béton armé exposée 27 mois à une corrosion naturelle en présence de cycles de mouillage-séchage d'un brouillard salin : début des expérimentations en 2010 d'après Yu et al. [6].

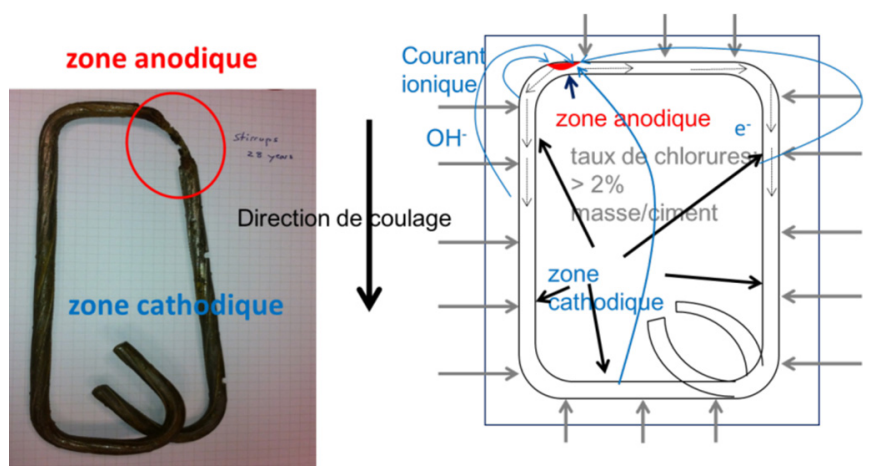

Fig. 3. Corrosion non uniforme d'un cadre d'effort tranchant d'une poutre en béton armé malgré un taux de chlorures identiques le long du périmètre du cadre (crédit photo: Raoul François)

outre, être capable de mesurer les vitesses de corrosion est la pierre angulaire de toutes les recherches sur les modèles de cinétique de corrosion (dans la phase de propagation de la corrosion). Mais pour les aciers d'armatures du béton armé, les réactions anodique et cathodique se produisent sur des zones spatialement séparées. Dans cette situation, l'applicabilité de la méthode traditionnelle, pour la mesure non destructive des taux de corrosion, dite de résistance de polarisation linéaire (LPR), est très limitée [11] et même inexacte [12]. En conséquence, il n'existe pas aujourd'hui de mesure fiable de la vitesse de corrosion.

La protection cathodique (PC) est une technique utilisée pour contrôler (en prévention ou en réparation) la corrosion d'un métal en le faisant fonctionner en tant que cathode dans une cellule électrochimique. La première application de la protection cathodique a été mise en œuvre par Davy, [13] pour empêcher la corrosion des revêtements en cuivre des navires de la marine britannique. Cette technique est également utilisée pour les pipelines, les jetées portuaires, les fondations par pieux, les plates-formes offshore et les structures métalliques en général. La PC peut aussi être appliquée à des structures existantes en béton armé (nommée effectivement protection cathodique), à de nouvelles structures de béton armé (nommée prévention cathodique) et a été bien décrite depuis quelques décennies [14-18]. Dans les structures en béton armé, ce sont les armatures de renforcement qui jouent le rôle de cathode, le béton est alors l'électrolyte et un système anodique est placé sur la surface du béton ou noyé dans la structure. Les anodes sacrificielles (aluminium, zinc) sont utilisées pour la protection cathodique galvanique (PCAG), tandis que des revêtements de titane activé Mixed Metal oxide (MMO) ou un revêtement électroconducteur connecté à un redresseur sont utilisés pour la protection cathodique à courant imposé (PCCI). Selon la norme EN ISO 12696 (2016) [19], les densités de courant à appliquer en prévention cathodique sont dans la gamme $0,2 \mathrm{~mA} / \mathrm{m}^{2}$ à $2 \mathrm{~mA} / \mathrm{m}^{2}$ de surface d'armature en acier, et dans la gamme de $2 \mathrm{~mA} / \mathrm{m}^{2}$ à $20 \mathrm{~mA} / \mathrm{m}^{2}$ de surface d'armature en acier pour la protection cathodique curative sur les structures existantes. On note $E_{O N}$ le potentiel des armatures sous courant de protection cathodique. Après coupure du courant de protection, les armatures se dépolarisent. La mesure effectuée immédiatement après la coupure (typiquement $<1 \mathrm{~s}$ ) est supposée s'affranchir de la chute ohmique (résistance électrique du béton) et est noté $E_{O F F}$.

La norme EN ISO 12696 précise que la protection contre la corrosion est assurée si l'une des trois conditions suivantes est vérifiée pour l'ensemble de la structure:

- un potentiel «Instant off» $\left(E_{O F F}\right)$ plus négatif que $-720 \mathrm{mV}$ par rapport à $\mathrm{Ag} / \mathrm{AgCl} / \mathrm{KCl} 0,5 \mathrm{M}$ (ce qui correspond à $-680 \mathrm{mV} / \mathrm{ECS}$ (électrode au calomel saturé)) ;

- une décroissance (dépolarisation) de potentiel d'au moins $100 \mathrm{mV}$ par rapport à $E_{O F F}$; sur un maximum de 24 heures;

- une décroissance (dépolarisation) de potentiel sur une longue période (généralement de $24 \mathrm{~h}$ ou plus) d'au moins $150 \mathrm{mV}$ par rapport à $E_{O F F}$ sous réserve d'une dépolarisation continue et de l'utilisation d'électrodes de référence (et non pas de sondes métalliques témoins) pour le suivi des mesures au-delà $24 \mathrm{~h}$.

De plus, l'EN ISO 16696 précise également d'autres limites: aucun potentiel «Instant off» $\left(E_{O F F}\right)$ moins 
négatif que $-900 \mathrm{mV}$ par rapport à $\mathrm{Ag} / \mathrm{AgCl} / \mathrm{KCl} 0,5 \mathrm{M}$, et aucun potentiel «Instant off» $\left(E_{O F F}\right)$ moins négatif que $-1100 \mathrm{mV}$ par rapport à $\mathrm{Ag} / \mathrm{AgCl} / \mathrm{KCl} 0,5 \mathrm{M}$, pour éviter respectivement un risque de fragilisation par l'hydrogène des aciers de précontraintes et un endommagement de l'interface acier-béton.

Le potentiel $E_{O F F}$ qui correspond au potentiel $E_{O N}$ avec déduction de la chute ohmique est le potentiel supposé réel, par la norme EN ISO 12696, pendant l'application de la protection cathodique, au niveau de l'acier. L'existence d'un gradient de potentiel, ne serait-ce que de $200 \mathrm{mV}$, (valeur minimale communément admise comme différence entre zones actives et passives) compromet l'obtention en tout point d'une valeur de $E_{\text {OFF }}$ inférieure à $-720 \mathrm{mV} / \mathrm{Ag} /$ $\mathrm{AgCl} / \mathrm{KCl} \quad 0,5 \mathrm{M}$ mais en aucun point inférieure à $-900 \mathrm{mV} / \mathrm{Ag} / \mathrm{AgCl} / \mathrm{KCl} 0,5 \mathrm{M}$ car l'écart n'est que de $180 \mathrm{mV}$ entre les deux critères. Le premier critère de l'EN ISO 12696 considère donc implicitement que le potentiel $E_{\text {OFF }}$ est uniforme dans toute la structure et il vise donc à déplacer l'acier dans son domaine d'immunité suivant le diagramme de Pourbaix [20].

Le critère de $100 \mathrm{mV}$ (dépolarisation) est basé sur une enquête de terrain menée en 1989 par Han et al. [21] sur 466 installations de protection cathodique en Amérique du Nord, où 223 structures ont été contrôlées en utilisant ce critère. Ce critère est également désigné dans la recommandation NACE, 1990 [22], qui préconise une période de temps de 4 heures. L'utilisation de ce critère pour les structures en béton armé a été également reprise dans une étude empirique réalisée par Bennett et Mitchell [23]. Mais ils recommandent d'augmenter la dépolarisation de 100 à $150 \mathrm{mV}$. Funahashi et Bushman [24] ont montré que des valeurs de dépolarisation entre 155 et $240 \mathrm{mV}$ sont nécessaires, en fonction de la teneur en chlorures du béton. D'autres études montrent qu'une dépolarisation de $100 \mathrm{mV}$ est trop élevée, et est significative d'une polarisation excessive de l'armature d'acier [25].

Il apparaît au final qu'aucune approche théorique n'existe pour justifier ces 3 critères d'efficacité de la protection dans la littérature relative à la PC. Par ailleurs, la connaissance usuelle du mécanisme de la protection cathodique est que la corrosion est atténuée en raison d'une diminution du gradient de potentiel dans le ferraillage (Fig. 4).

Ce point est très important par rapport à la vérification des critères : en effet, s'ils sont vérifiés en quelques points, ils peuvent ainsi être généralisés à l'ensemble de la structure. Mais si le processus de corrosion non uniforme avec des potentiels anodique et cathodique distincts conduit également sous PCCI à des potentiels distincts: cela rend impossible la vérification «en tout point» des critères de protection de la norme EN ISO 12696.

Il est important de noter que la communauté de la Protection Cathodique du secteur Terre, travaillant dans le domaine des structures métalliques enterrées, est consciente depuis des décennies de l'hétérogénéité du champ de potentiel en présence de corrosion par macro-piles [27].

Une étude expérimentale et numérique d'un système de protection cathodique par courant imposé est réalisée sur un mur en béton armé. La combinaison de ce cas test expérimental et de sa simulation numérique permet de
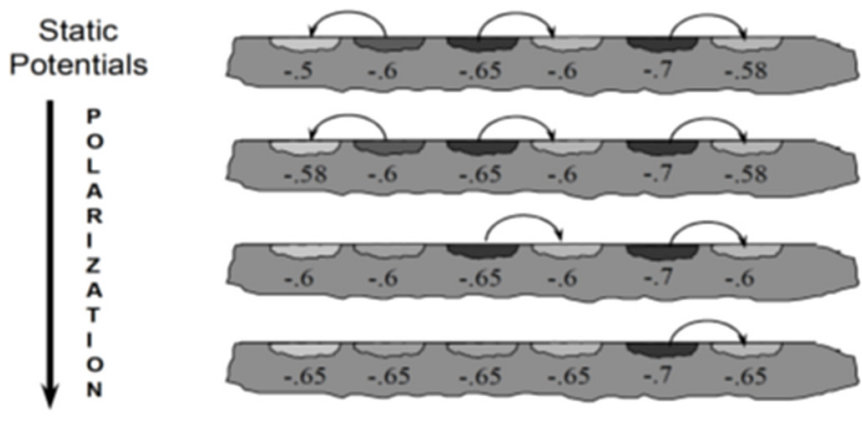

Corrosion Mitigated

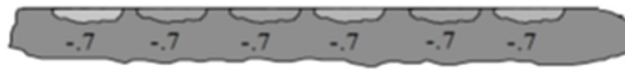

Fig. 4. Illustration du principe de la protection cathodique d'après NACE 2000 [26].

discuter les critères de fonctionnement et de performance définis dans la norme En ISO 12696.

\section{Programme expérimental : essais et méthodes}

L'étude expérimentale du système de protection cathodique a été réalisée sur un mur en béton armé nommé M3PC (Fig. 5) de hauteur $75 \mathrm{~cm}$, de largeur $100 \mathrm{~cm}$ et d'épaisseur $20 \mathrm{~cm}$. Le béton utilisé est un béton ordinaire dont la composition par $\mathrm{m}^{3}$ est la suivante: $52,5 \mathrm{~kg}$ de ciment CEM I, $165 \mathrm{~kg}$ de gravier, $105 \mathrm{~kg}$ de sable et $30 \mathrm{~L}$ d'eau. Le démoulage du mur a été réalisé après 5 jours et le ferraillage du mur est constitué de 5 barres horizontales et de 4 barres verticales d'armatures à Haute Adhérence de diamètre $8 \mathrm{~mm}$, avec des enrobages respectifs de 30 et $40 \mathrm{~mm}$. Les barres sont non connectées électriquement. Une zone de corrosion locale a été initiée par migration de chlorures dans une zone du mur. Le système de migration consistait en 2 coudes en PVC de diamètre $100 \mathrm{~mm}$, collés de chaque coté du mur. Un coude étant rempli d'une solution à $0,5 \mathrm{M}$ de $\mathrm{NaCl}$ et le second d'une solution à $0,5 \mathrm{M}$ de $\mathrm{NaOH}$. Une différence de potentiel de $5 \mathrm{~V}$ a été imposée entre les deux solutions, avec des électrodes en titane activé MMO, jusqu'à initiation de la corrosion des barres les plus proches du coude rempli de $\mathrm{NaCl}$. L'activité de corrosion a été vérifiée par l'apparition d'un courant galvanique entre la barre verticale V3 et le reste des armatures. Ainsi, seule une zone restreinte du mur est contaminée par les chlorures. Par ailleurs, le ferraillage n'a pas été soumis à une polarisation significative puisque les armatures n'ont pas été utilisées comme électrodes dans le processus de migration. L'application de la protection cathodique se fait par une anode surfacique constituée d'un treillis titane activé MMO : le treillis est installé sur la face arrière et recouvert d'une couche de mortier de ciment CEM I. L'installation sur la face arrière du mur présente l'intérêt de pouvoir mesurer le potentiel des armatures par rapport à une électrode de référence placée en surface du béton, sur la face par laquelle est arrivée la contamination par les chlorures, ce qui n'est pas en général le cas dans les ouvrages réels ou seule la mesure de potentiel des armatures par rapport à une électrode de référence interne est 


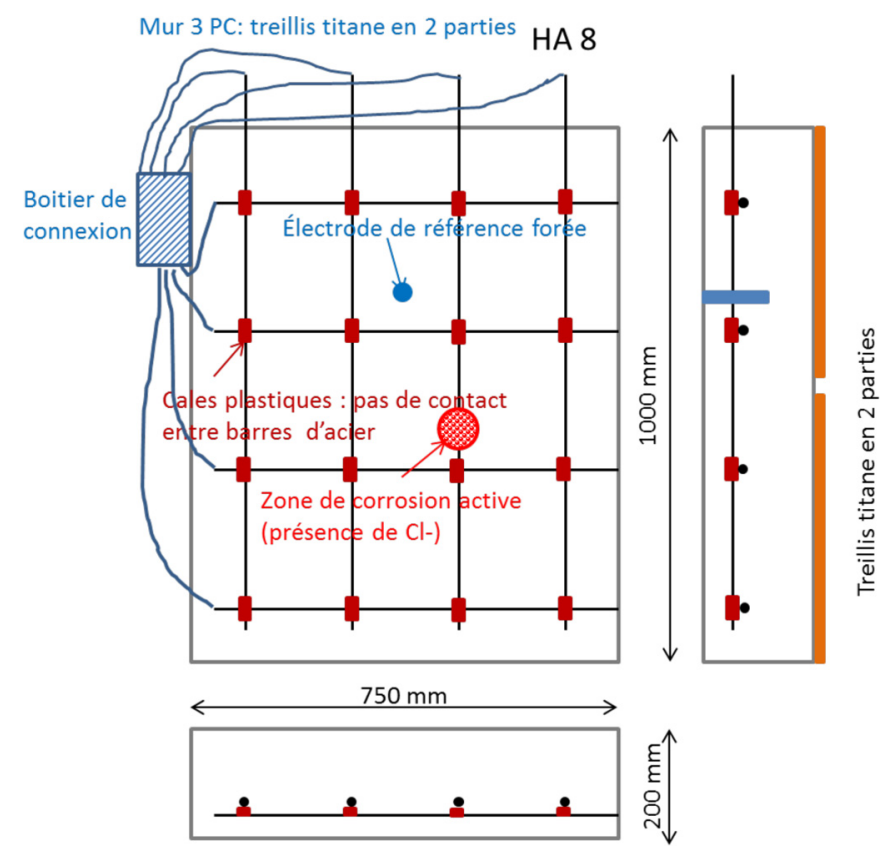

Fig. 5. Mur M3PC en béton armé avec armatures isolées électriquement par des manchons à chaque intersection et équipé d'un treillis titane activé MMO.

possible. Le mur est aussi équipé d'une électrode interne FORCE ERE 20 au manganèse. À noter que les armatures sont toutes déconnectées électriquement les unes des autres, et que la connexion électrique peut être établie par un boîtier extérieur avec ou sans interconnexion d'un ampèremètre. Ce dispositif permet ainsi d'avoir accès aux courants de corrosion entre les diverses armatures, ce qui représente une approche, certes simplifiée mais réelle, des courants de corrosion de macro-piles dans le mur. En effet, le bémol est qu'on n'a pas accès aux courants de macropiles qui existent sur une même barre.

Pour caractériser les paramètres électrochimiques qui seront utilisés dans la partie simulation numérique, il faut avoir accès séparément au comportement de l'acier passif, (qui jouera le rôle de cathode) et celui de l'acier actif, (qui jouera le rôle d'anode). Dans ce but, des éprouvettes cylindriques en béton de $100 \mathrm{~mm}$ de diamètre et $100 \mathrm{~mm}$ de hauteur contenant un acier HA centré de diamètre $12 \mathrm{~mm}$ et de longueur $30 \mathrm{~mm}$ ont été coulées. La surface d'acier correspond donc à $1131 \mathrm{~mm}^{2}$ ou $11,3 \mathrm{~cm}^{2}$. L'acier est inséré dans l'éprouvette de béton sans aucun traitement, il est ainsi «tel que reçu», c'est-à-dire recouvert avant coulage par une couche de calamine liée au processus de fabrication des aciers. Après coulage, les réactions de la solution basique du béton avec la couche de calamine conduisent à la formation d'une couche de passivation, cette éprouvette sera celle permettant d'évaluer le comportement à la zone cathodique du processus de corrosion (éprouvette passive). Une autre éprouvette a été exposée à une solution chlorée à $35 \mathrm{~g} / \mathrm{L}$ de $\mathrm{NaCl}$ après séchage à $50 \%$ d'HR pour obtenir une pénétration de chlorures par succion capillaire et ainsi une dépassivation de l'acier. Cette éprouvette permettra d'évaluer le comportement à la zone anodique du processus de corrosion (éprouvette active).

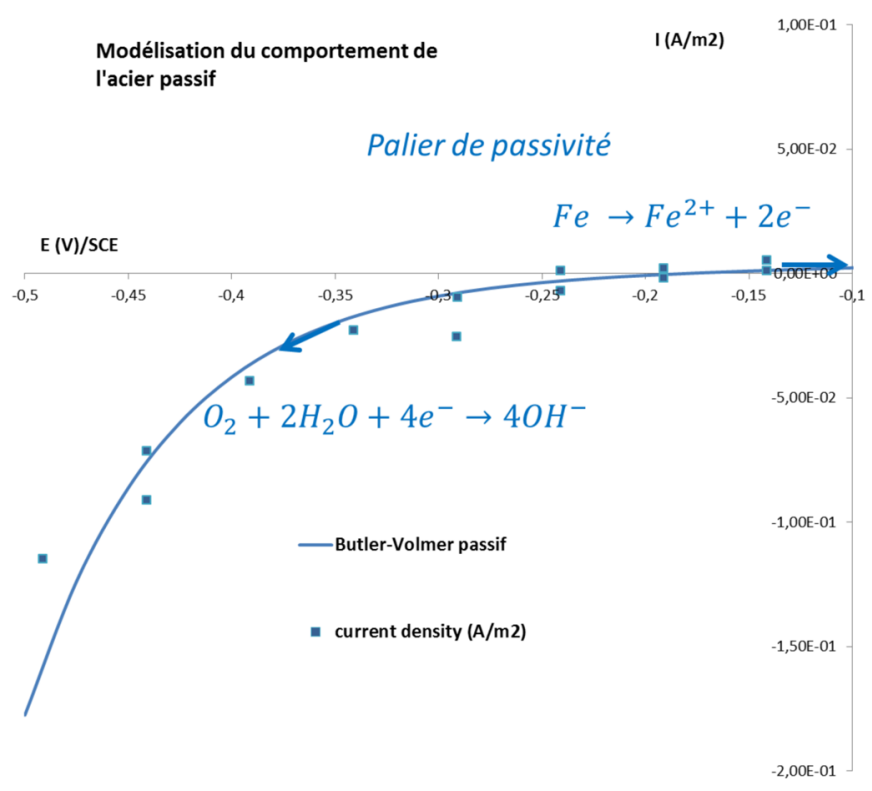

Fig. 6. Comportement électrochimique de l'acier à l'état passif avec visualisation du palier de passivité, et modélisation par la relation de Bultler-Volmer.

\section{4 Étude numérique: principe et méthodes}

Un système de corrosion non uniforme résulte de la connexion entre une zone anodique et une zone cathodique. En mode couplé, la zone anodique correspond à l'oxydation du fer (ou zone active de corrosion) et la zone cathodique à la réduction de l'oxygène (ou zone passive) pour le cas du béton armé aérien. En mode découplé, il s'agit de deux systèmes de corrosion uniforme.

Le comportement d'un acier passif ou actif dans un béton aérien correspond au couplage de 2 électrodes réversibles : oxydation du fer pour la partie anodique (Eq. (1)) et réduction de l'oxygène pour la partie cathodique (Eq. (2)).

Équation (1) : oxydation du fer pour la partie anodique:

$$
\mathrm{Fe} \rightarrow \mathrm{Fe}^{2+}+2 \mathrm{e}^{-}
$$

Équation (2): réduction de l'oxygène pour la partie cathodique :

$$
\mathrm{O}_{2}+2 \mathrm{H}_{2} \mathrm{O}+4 \mathrm{e}^{-} \rightarrow 4 \mathrm{OH}^{-}
$$

Le couplage des deux électrodes réversibles dans le béton sain donne lieu à un comportement passif de l'acier, c'est-à-dire avec la présence d'un palier anodique avec une densité de courant très faible pour une grande plage de potentiel. Il doit être noté ici que l'acier passif peut être considéré comme un système de corrosion uniforme [12], bien que le courant de corrosion soit très faible et souvent négligé. Le comportement de polarisation de tels systèmes peut être modélisé par l'équation de Butler-Volmer (Eq. (3)), en utilisant des ensembles appropriés pour les paramètres électrochimiques. Le comportement de l'acier est caractérisé par un essai de polarisation (Fig. 6) qui ne correspond pas à l'essai classique de polarisation linéaire 
(LPR), car aucune linéarisation du comportement n'est réalisée. De plus, étant donné l'influence non négligeable de la vitesse de balayage sur les densités de courant anodiques et cathodiques $[12,28]$, les courbes de polarisation sont tracées en régime quasi stationnaire point par point avec un incrément de $50 \mathrm{mV}$ sur une plage de $\pm 300 \mathrm{mV}$ et attente de 400 s avant de relever la valeur mesurée. En effet, des mesures préliminaires ont montré que le courant était quasiment établi à son régime permanent après $400 \mathrm{~s}$ d'attente.

Il peut être utile, pour les non-spécialistes de la corrosion, de rappeler ici que l'utilisation de l'équation de Butler-Volmer ne signifie pas une réversibilité des réactions anodiques et cathodiques prises séparément: ainsi par exemple la branche cathodique ne correspond pas à la formation de $\mathrm{Fe}$ mais à la réduction du dioxygène et la branche anodique ne correspond pas à la création de dioxygène mais l'oxydation du fer.

$$
\begin{aligned}
j_{n}= & j_{\text {corr }}\left(\exp \left(\frac{\ln (10)\left(E-E_{\text {corr }}\right)}{b_{a}}\right)\right. \\
& \left.-\exp \left(-\frac{\ln (10)\left(E-E_{\text {corr })}\right)}{b_{c}}\right)\right) .
\end{aligned}
$$

$E_{\text {corr }}$ est le potentiel de corrosion libre $(\mathrm{V} / \mathrm{ref}) \mathrm{du}$ système de corrosion à l'équilibre; $j_{n}$ est la densité de courant net $\left(\mathrm{A} / \mathrm{m}^{2}\right)$ circulant à travers l'interface metal-electrolyte du système uniforme forcé au potentiel $E$ (impliquant une polarisation par rapport au potentiel d'équilibre $\left.E_{\text {corr }}\right) ; j_{\text {corr }}$ est la densité de courant de corrosion $\left(\mathrm{A} / \mathrm{m}^{2}\right)$, ce qui correspond à l'échange de courant du système de la corrosion uniforme; $b_{a}$ et $b_{c}$ sont respectivement les coefficients de Tafel anodique et cathodique ( $\mathrm{V} /$ décade) du système électrochimique.

En présence de $\mathrm{Cl}^{-}$ou de $\mathrm{CO}_{2}$ ayant carbonaté le béton, le comportement de l'acier devient localement actif avec une réponse anodique beaucoup plus raide. La figure 7 présente les courbes de polarisation expérimentales des éprouvettes cylindriques actives et passives présentées dans la partie expérimentale. La corrosion localisée se définit comme le couplage électrique de deux électrodes spatialement séparées. On peut aussi l'appeler corrosion galvanique même s'il s'agit d'un même métal car ces électrodes sont constituées par les 2 systèmes de corrosion distincts (actif et passif). Cette définition implique que la distance entre les 2 électrodes ne permet plus de négliger les effets ohmiques dus à la résistivité de l'électrolyte (béton). Les sites anodiques et cathodiques étant distants, ils ne peuvent se rejoindre au niveau d'un potentiel commun et il s'en suit une différence significative entre potentiels anodique $\left(E_{a}\right)$ et cathodique $\left(E_{p}\right)$, un gradient de potentiel dans le volume et un courant de volume, dit courant galvanique ou courant de macro-piles $I_{m}$ (Fig. 7).

L'écriture de l'équilibre d'un système de macro-piles (ou galvanique) nécessite le recours à une équation complémentaire, en l'occurrence la loi d'Ohm. En effet, l'équilibre d'un tel système dépend naturellement de ses composantes électrochimiques, mais également de la résistivité électrique de l'électrolyte et de la structure

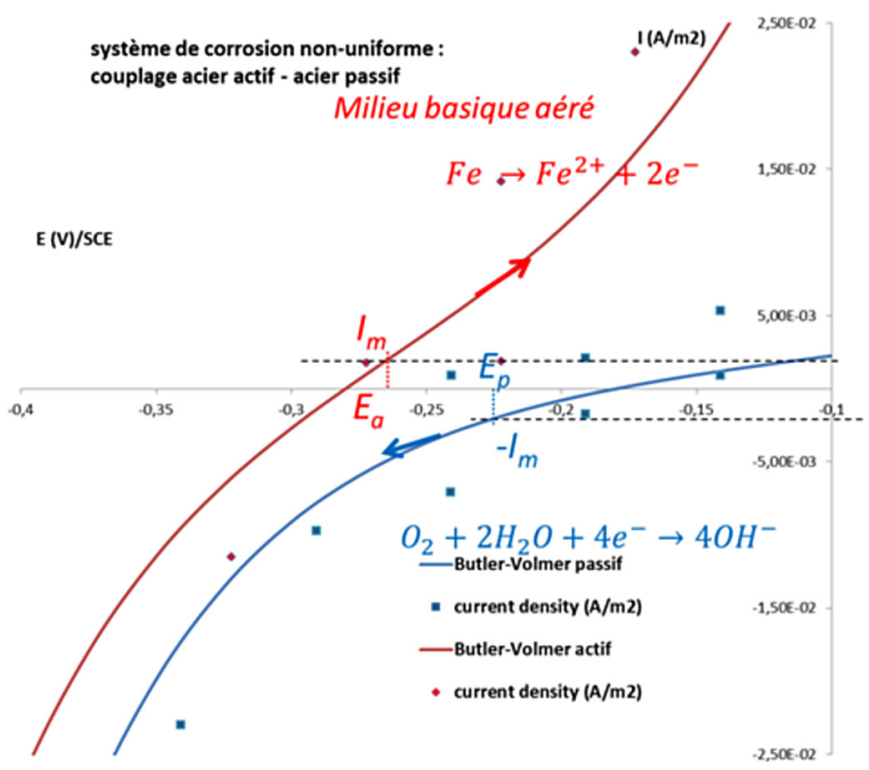

Fig. 7. Comportement expérimental des éprouvettes actives et passives. Visualisation qualitative et simplifiée (1D) de la polarisation réciproque de sites actifs (courbe rouge) et passifs (courbe bleu) : la zone active de corrosion se déplace alors sur sa branche anodique et la zone passive sur sa branche cathodique pour atteindre respectivement, le point de coordonnées $\left(E_{a}, I_{m}\right)$ et le point de coordonnées $\left(E_{p},-I_{m}\right) . I_{m}$ représente le courant de macro-pile et $E_{a}$ et $E_{p}$ sont représentatifs du gradient de potentiel dans l'élément de béton armé.

Tableau 1. Paramètres de Butler-Volmer acier actif et acier passif.

\begin{tabular}{lcc}
\hline & Passif & Actif \\
\hline$E_{\text {corr }}(\mathrm{V})$ & $-0,14$ & $-0,28$ \\
$i_{\text {corr }}\left(\mathrm{A} / \mathrm{m}^{2}\right)$ & 0,001 & 0,005 \\
$b_{a}$ & 0,4 & 0,2 \\
$b_{c}$ & 0,16 & 0,16 \\
\hline
\end{tabular}

spatiale du système. À l'équilibre, un courant galvanique d'intensité $I_{m}$ circule dans le volume depuis les sites anodiques vers les sites cathodiques (Fig. 5).

Les paramètres de BV issus des courbes expérimentales sont donnés dans le tableau 1.

L'étude numérique consiste à étudier successivement le système de corrosion libre (avant protection cathodique) puis son évolution en fonction de l'intensité du courant de protection cathodique. On utilise le code d'élément finis COMSOL Multiphysics ${ }^{\circledR}$ avec les conditions limites à l'interface acier-béton correspondant au comportement électrochimique des zones actives et passives (paramètres de BV). La corrosion a été simulée principalement sur les barres horizontales 3 et 4 et un peu sur la barre verticale 3 (zones bleues dans la Fig. 8) pour tenir compte du point contamination central par les chlorures.

Pour un système de corrosion localisée $3 \mathrm{D}$, le système d'équation de BV précédent est simplement reformulé sous 


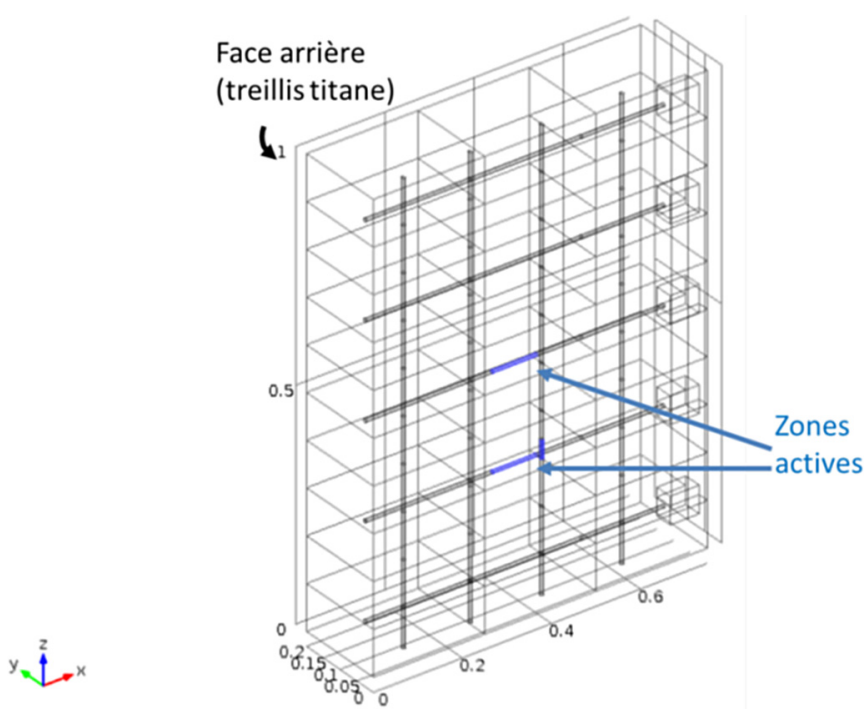

Fig. 8. Modèle géométrique pour le mur en béton armé avec localisation des zones corrodées.

la forme d'un problème différentiel aux limites, considérant des champs de potentiels anodiques et cathodiques au lieu de valeurs scalaires, la loi d'Ohm locale et l'équation de conservation de la charge électrique (Eqs. (4) et (5)). Ce calcul peut donc se faire avec un code d'éléments finis tel que COMSOL dans une formulation «électrique» et des conditions limites d'interface acier-béton correspondant aux relations de BV.

Équation (4) : loi d'Ohm locale, transport des charges :

$$
j=-\frac{1}{\rho} \nabla E,
$$

Équation (5): conservation de la charge:

$$
\nabla \cdot j=0 .
$$

\section{Résultats expérimentaux}

Les cartographies de potentiel à haute résolution ont été réalisées à l'aide d'un multimètre Fluke relié à une électrode au calomel saturé. On a utilisé un maillage carré de $1,5 \mathrm{~cm}$, ce qui entraîne 3365 points de mesure. Les cartes de potentiel ont été tracées avec le logiciel Surfer $7^{\circledR}$. Deux étapes sont réalisées: une première, où l'on cherche le courant de protection qui annule le courant de corrosion entre les différentes armatures du mur en béton armé, une seconde où conformément à la norme EN ISO 12696:2016, un courant de protection correspondant à $15 \mathrm{~mA} / \mathrm{m}^{2}$ d'acier a été appliqué.

Toutes les barres d'acier sont reliées électriquement par le boîtier de connexion (Fig. 5). Avant l'application du courant de protection, le potentiel libre mesuré par l'électrode interne était de $-430 \mathrm{mV} / \mathrm{ECS}$.

La figure 9 présente la cartographie de potentiel naturel (libre) mesurée sur la face avant du mur en béton armé. La zone de corrosion induite par la migration des chlorures au

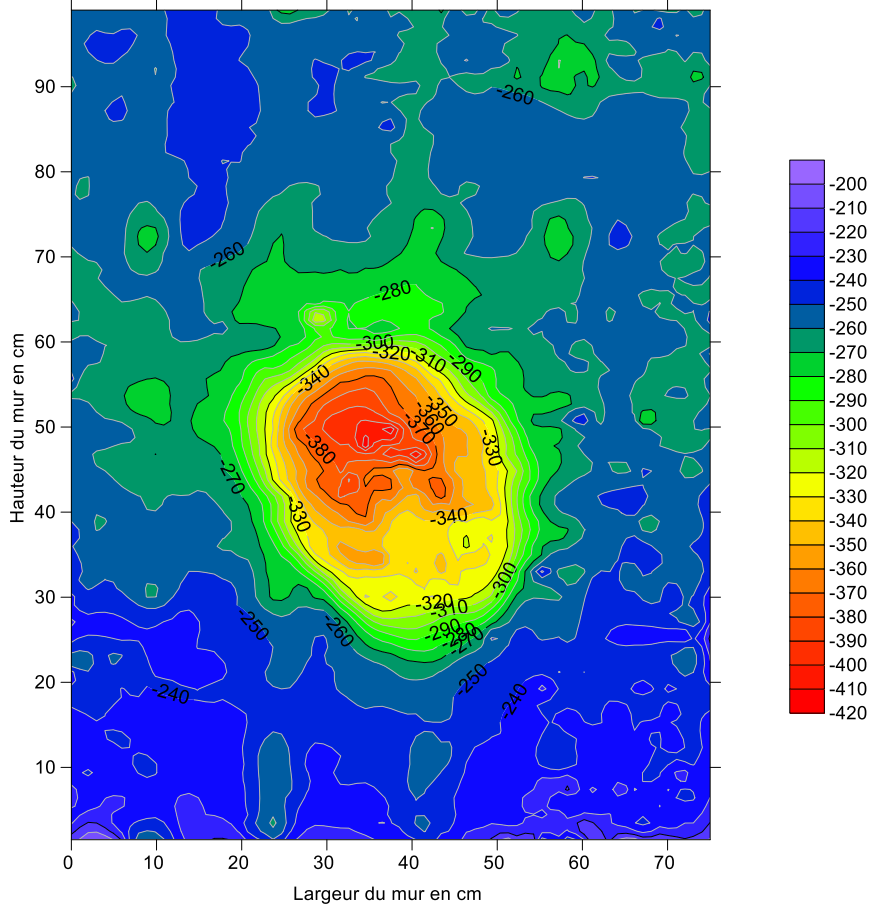

Fig. 9. Cartographie de potentiel libre (mV/ECS).

centre de la paroi peut être clairement observée sur la carte de potentiel. La figure 10 présente la cartographie de potentiel interprétée en valeur relative par la méthode exposée par François et al. en 1990 et 1994 [29,30]. Il s'agit de s'affranchir des effets de chute ohmique et donc de la variabilité des mesures en fonction de l'humidité ambiante et de l'humidité du béton, en faisant une interprétation des différences par rapport à la moyenne des valeurs absolues des mesures sur une zone donnée. La figure 10 met clairement en évidence la zone de corrosion active, et le reste du ferraillage qui est passif.

Dans un premier temps, on s'intéresse au courant de protection permettant de stopper la corrosion. Il est compliqué voire impossible aujourd'hui de mesurer le courant de corrosion d'un système de corrosion non uniforme comme rappelé dans l'introduction. Une approche (sans doute discutable) basée sur la mesure du courant de corrosion entre barres actives et passives a été utilisée : les barres horizontales $\mathrm{n}^{\circ} 3$ et $\mathrm{n}^{\circ} 4$ (en notant $\mathrm{n}^{\circ} 1$ la barre supérieure) sont celles qui sont exposées aux chlorures et donc potentiellement actives. En connectant via un potensiostat en mode ZRA ou un ampèremètre l'une ou l'autre de ces barres au reste du réseau d'armatures, on aura accès au courant de corrosion «galvanique» entre l'une ou l'autre des barres et le reste des aciers passifs. Cela nous donnera un ordre de grandeur du courant de corrosion, sachant que dans cette opération, on n'a pas accès au courant de corrosion de macro-piles entre la partie corrodée de la barre 3 ou 4 (Fig. 5) et le reste de la barre 3 ou 4 qui est encore passif. La mesure donne 54 microA entre la barre 3 et le reste du ferraillage et 16 microA pour la barre 4. La barre 3 qui est la plus affectée par la corrosion va servir de référence pour «annuler » le courant de corrosion. 

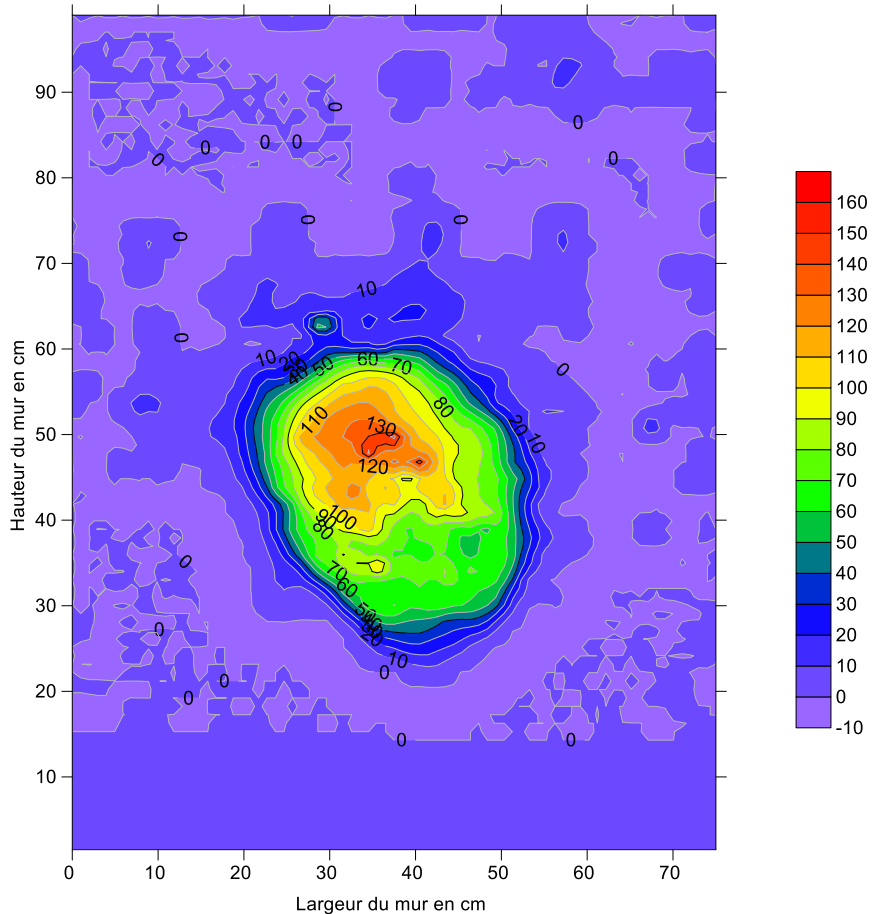

Fig. 10. Cartographie de potentiel libre en valeur absolue de l'écart positif par rapport à la moyenne $(\mathrm{mV} / \mathrm{ECS})$ : d'après François et al. (1990, 1994).

On applique donc un courant de protection sur le treillis et la valeur du courant de corrosion mesuré entre la barre 3 et le reste du ferraillage diminue et s'annule pour une valeur du courant de protection de $0,3 \mathrm{~mA}$, ce qui correspond à une densité de courant imposé de $1,5 \mathrm{~mA} / \mathrm{m}^{2}$ de surface d'acier.

Le potentiel mesuré par l'électrode de référence interne est quant à lui voisin de $-500 \mathrm{mV} / \mathrm{ECS}$ (le potentiel libre était de $-430 \mathrm{mV} / \mathrm{ECS}$ )

On est donc certain qu'à ce degré de polarisation aucun des 3 critères de la norme n'est vérifié : on n'atteint pas les $-680 \mathrm{mV} / \mathrm{ECS}$ (en instant «OFF») puisqu'on est déjà supérieur en «ON» et la polarisation est inférieure à $100 \mathrm{mV}$ donc on ne pourra donc pas obtenir une dépolarisation de $100 \mathrm{mV}$ et a fortiori de $150 \mathrm{mV}$. On a pourtant stoppé le courant de corrosion mesuré entre la barre active 3 et le reste du ferraillage. Ce résultat indique que les critères de la norme EN ISO 12696 sont sans doute très conservatifs.

Dans un second temps, on se place dans les conditions recommandées par la norme EN ISO 12696 en appliquant une densité de courant de corrosion de $15 \mathrm{~mA} / \mathrm{m}^{2}$, ce qui correspond à une intensité de $3 \mathrm{~mA}$. Une fois le système de PCCI en marche, le potentiel mesuré par l'électrode interne a diminué et s'est stabilisé à $-679 \mathrm{mV} / \mathrm{ECS}$ après 1 semaine de polarisation.

La figure 11 présente la carte de potentiel sous PCCI après 50 jours de polarisation. On peut noter qu'il est rare d'avoir accès à la carte de potentiel des armatures sous PCCI car la surface exposée au processus de corrosion est en général celle qui reçoit la protection cathodique et est donc seulement représentative du potentiel anodique.

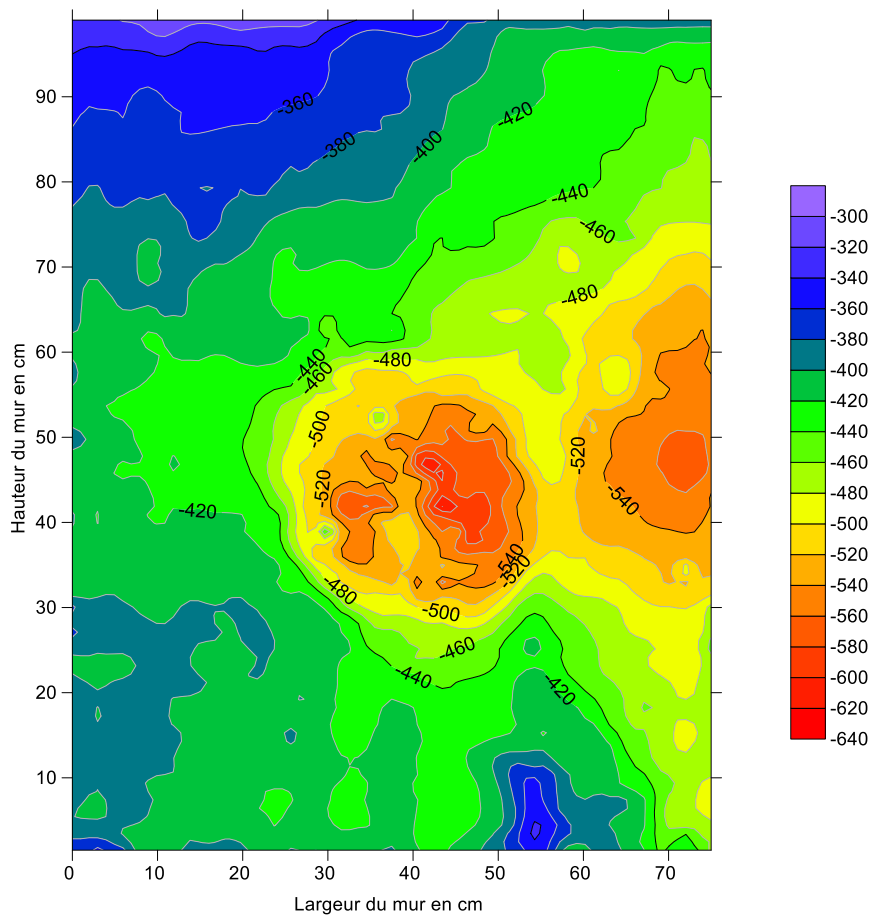

Fig. 11. Cartographie de potentiel ON sous PCCI (mV/ECS).

La carte de potentiel a globalement évoluée vers des valeurs plus électronégatives. Chaque point de mesure est plus négatif, mais le champ de potentiel sous polarisation reste non uniforme sur la surface du mur en béton. En fait, l'écart de potentiel maximal sur la surface du mur reste du même ordre de grandeur avant et pendant la polarisation : $220 \mathrm{mV}$ et $240 \mathrm{mV}$. Ce résultat indique que le potentiel à l'interface acier-béton n'est pas uniforme. La figure 12 montre la carte de potentiel tracé en écart par rapport à la moyenne des potentiels sur le mur. On constate que le puits de potentiel autour de la zone corrodée est le même avant et après polarisation, le puits est même légèrement accentué en intensité après polarisation. On a donc très clairement un champ de potentiel qui est déplacé vers des valeurs plus négatives par la protection cathodique mais pas de tendance à une uniformisation des potentiels. La seule différence entre les cartes de potentiels libre et sous polarisation cathodique est une tendance à avoir des potentiels plus négatifs sur le bord droit du mur.

On a réalisé également des cartographies de potentiel du côté du treillis anodique mais avec un maillage beaucoup plus grossier: la maille est de $18,75 \mathrm{~cm}$ sur la largeur et $25 \mathrm{~cm}$ sur la hauteur.

Les aciers de renforcement se trouvent à presque $20 \mathrm{~cm}$ de distance par rapport à la surface de mesure, le champ de potentiel en surface doit donc être moins représentatif du champ de potentiel au niveau des aciers que celui mesuré du côté opposé, à la fois en termes de gradient de potentiel et de valeurs absolues. La figure 13 montre effectivement que l'écart de potentiel est moins important que celui mesuré sur la surface exposée mais on remarque quand même un puits de potentiel sur la partie centrale du mur qui correspond donc à l'existence de la zone corrodée. 


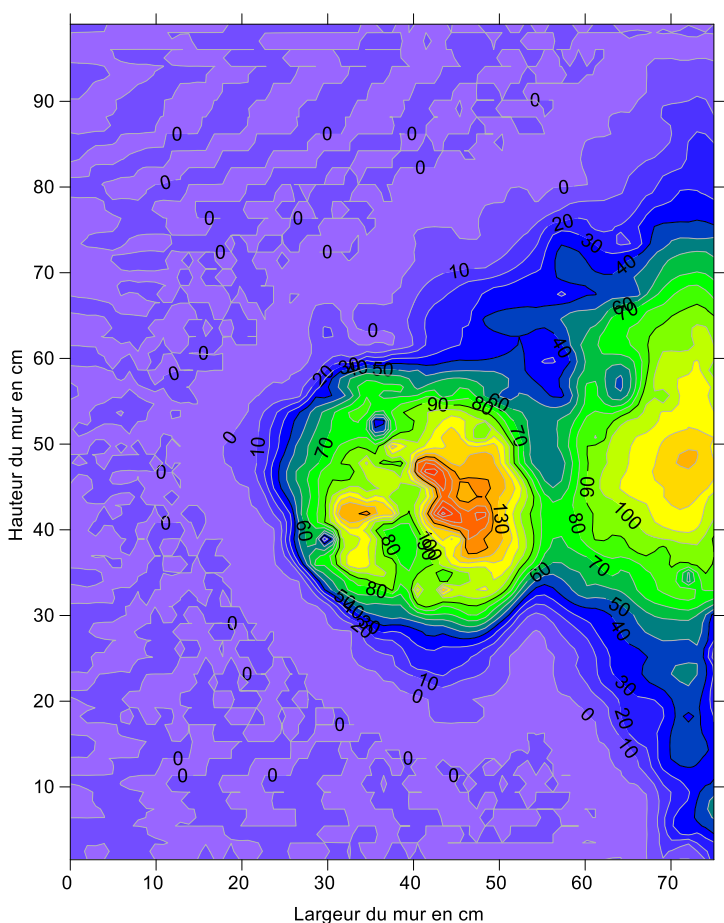

Fig. 12. Cartographie de potentiel ON sous PCCI (mV/ECS) en valeur absolue de l'écart positif par rapport à la moyenne.

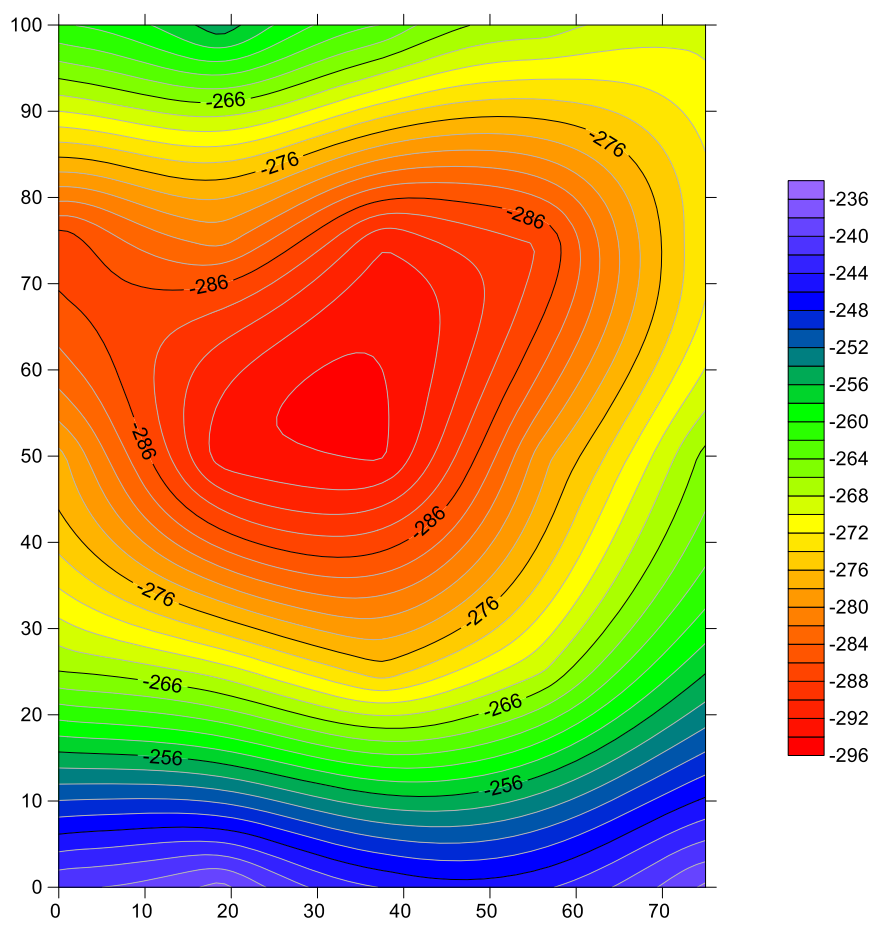

Fig. 13. Cartographie de potentiel libre du côté de l'ANODE $(\mathrm{mV} / \mathrm{ECS})$.

La figure 14 montre la cartographie de potentiels «ON» sous polarisation cathodique. Le treillis reçoit le courant et son potentiel est donc abaissé de façon significative jusqu'à des valeurs de $-1,1 \mathrm{mV} /$ ECS. Il est normal que sous cette polarisation, on ne puisse plus

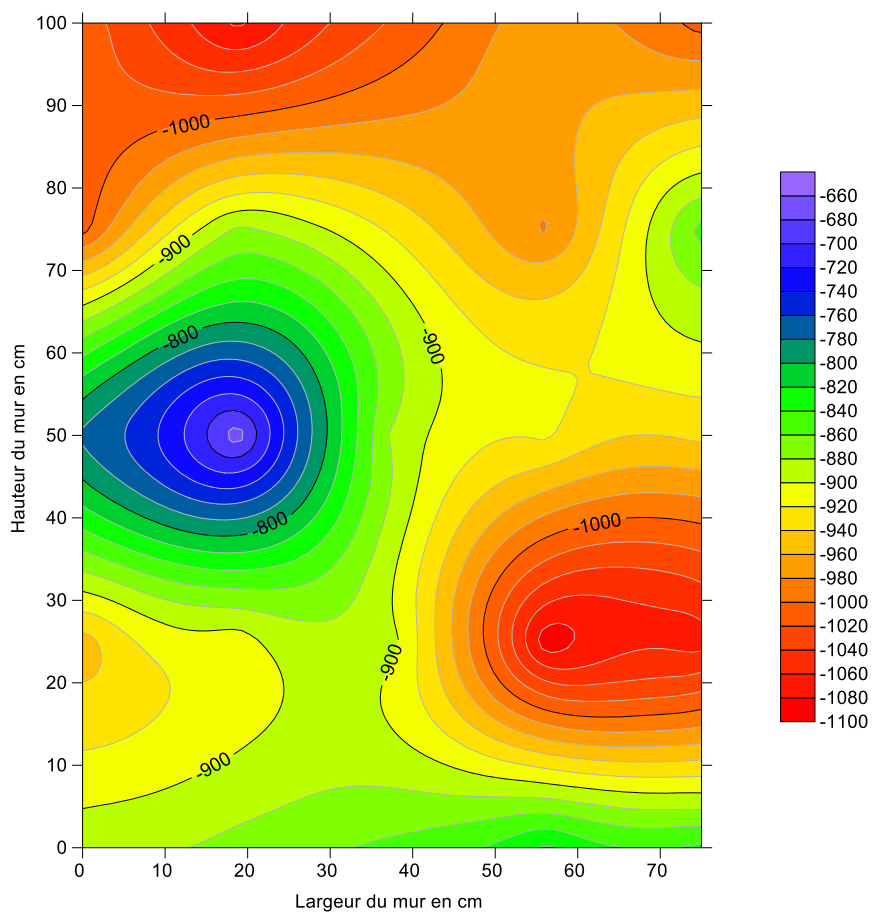

Fig. 14. Cartographie de potentiel ON sous PCCI du côté de l'ANODE (mV/ECS).

distinguer le champ de potentiel proche des armatures: ainsi sur site, on n'a généralement pas accès au champ de potentiel des armatures sous polarisation car la surface exposée est pratiquement toujours celle qui reçoit le support anodique pour le courant imposé, et on obtient donc des valeurs très négatives liées au courant de protection injecté.

Un résultat remarquable de cette mesure est que le champ de potentiel est très loin d'être uniforme. Il y a plus de $400 \mathrm{mV}$ d'écart sur la surface du mur. Il est probable qu'une application in situ de la PCCI soit au moins aussi non uniforme que celle obtenue sur ce mur en béton armé de petite dimension. Cela complique la modélisation du fonctionnement du système de corrosion sous protection cathodique car il n'est pas possible d'envisager une condition limite simple avec un champ de potentiel uniforme en surface.

On constate dans la figure 14 que le potentiel du côté treillis est beaucoup plus négatif vers le côté droit du mur, cela explique pourquoi le champ de potentiel «ON » sous polarisation mesuré sur la surface exposée (Figs. 11 et 12) présente un puits de potentiel qui déborde de la zone corrodée vers le côté droit du mur. On mesure donc en surface la composition du champ de potentiel résultant de la corrosion non uniforme avec le champ de potentiel résultant de l'imposition du courant de protection, lui aussi non uniforme.

Les figures 15 et 16 présentent les cartes de potentiel après respectivement $24 \mathrm{~h}$ et 7 jours de dépolarisation. Après $24 \mathrm{~h}$-dépolarisation, on peut observer que seule la zone corrodée au centre du mur est visible sur la carte, et qu'il en est de même au bout de 7 jours de dépolarisation (Figs. 17 et 18), et que cette zone de corrosion apparaît 


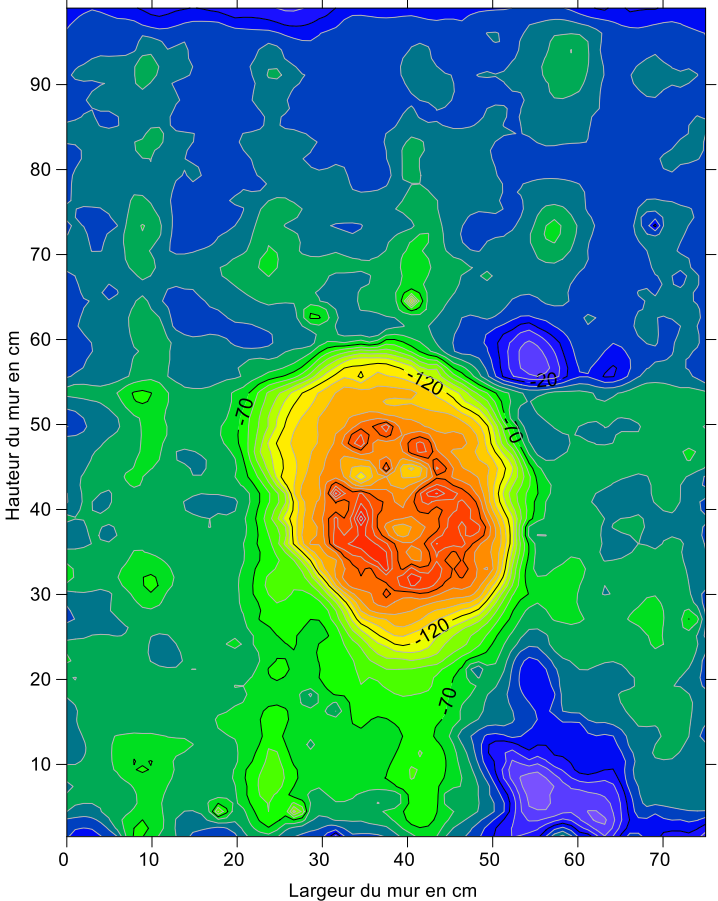

Fig. 15. Cartographie de potentiel OFF après $24 \mathrm{~h}$ de dépolarisation $(\mathrm{mV} / \mathrm{ECS})$.

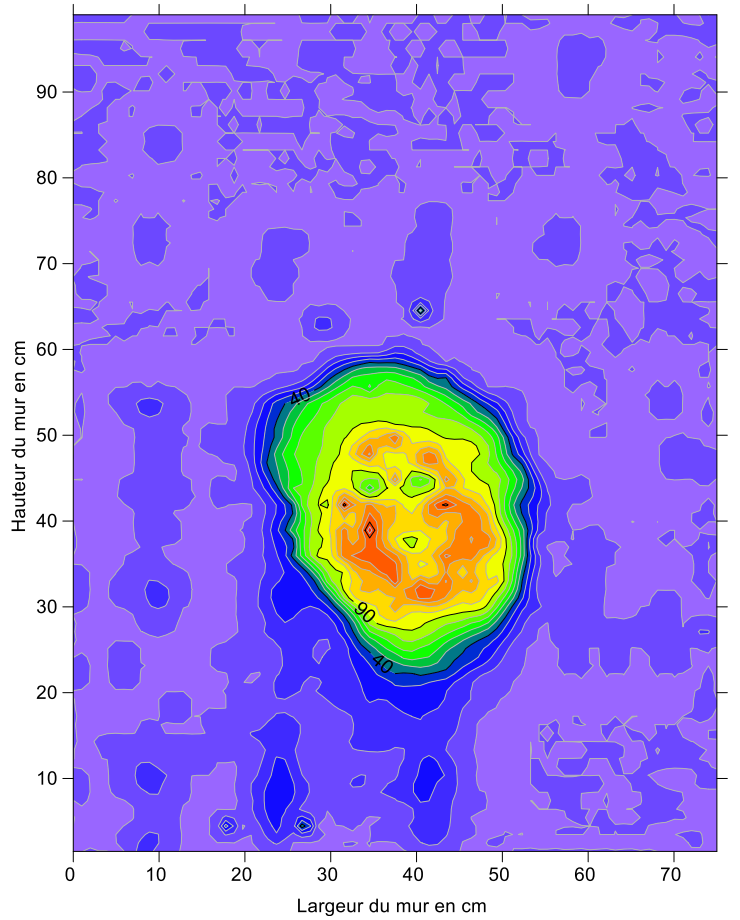

Fig. 16. Cartographie de potentiel OFF après $24 \mathrm{~h}$ de dépolarisation $(\mathrm{mV} / \mathrm{ECS})$ en valeur absolue de l'écart positif par rapport à la moyenne.

entourée d'une zone de potentiel dans le reste du mur presque uniforme. On note que les valeurs de potentiels après les cycles de polarisation et de dépolarisation qui ont duré presque 2 mois ont remonté de façon significative en

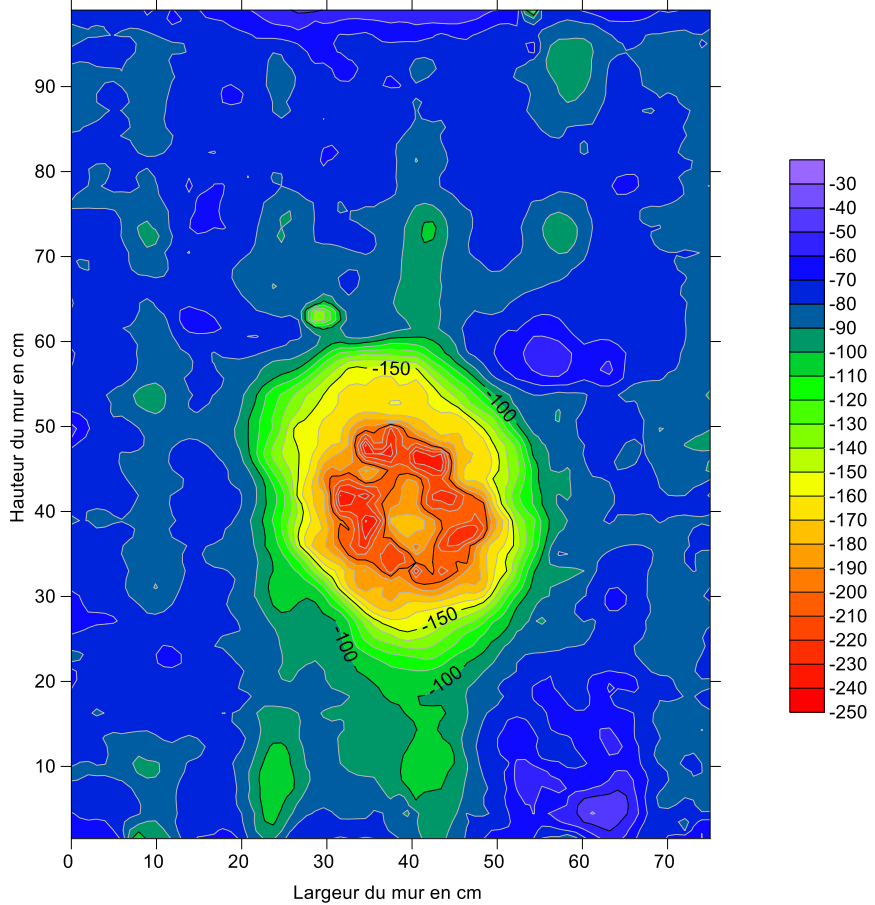

Fig. 17. Cartographie de potentiel OFF après $7 \mathrm{j}$ de dépolarisation $(\mathrm{mV} / \mathrm{ECS})$.

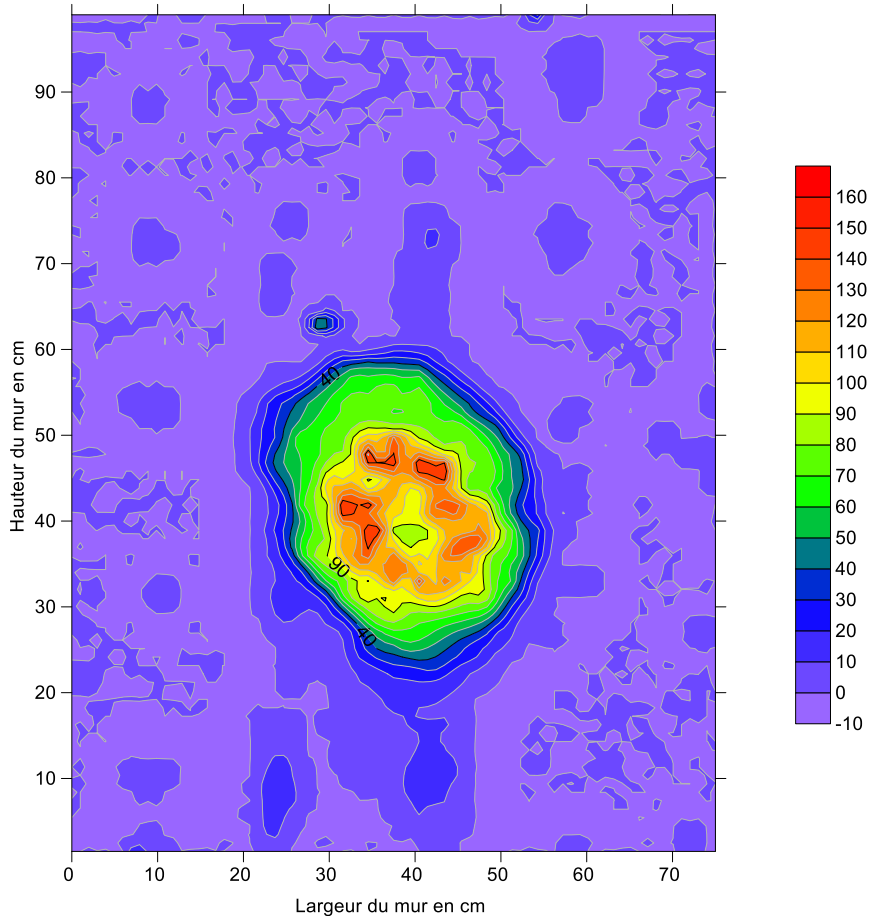

Fig. 18. Cartographie de potentiel OFF après $7 \mathrm{j}$ de dépolarisation $(\mathrm{mV} / \mathrm{ECS})$ en valeur absolue de l'écart positif par rapport à la moyenne.

raison du séchage du mur qui se trouve dans une salle de TP climatisée à $21^{\circ} \mathrm{C}$.

Il est donc remarquable de noter que les cartes de potentiel exprimées en valeur absolue de l'écart positif par 


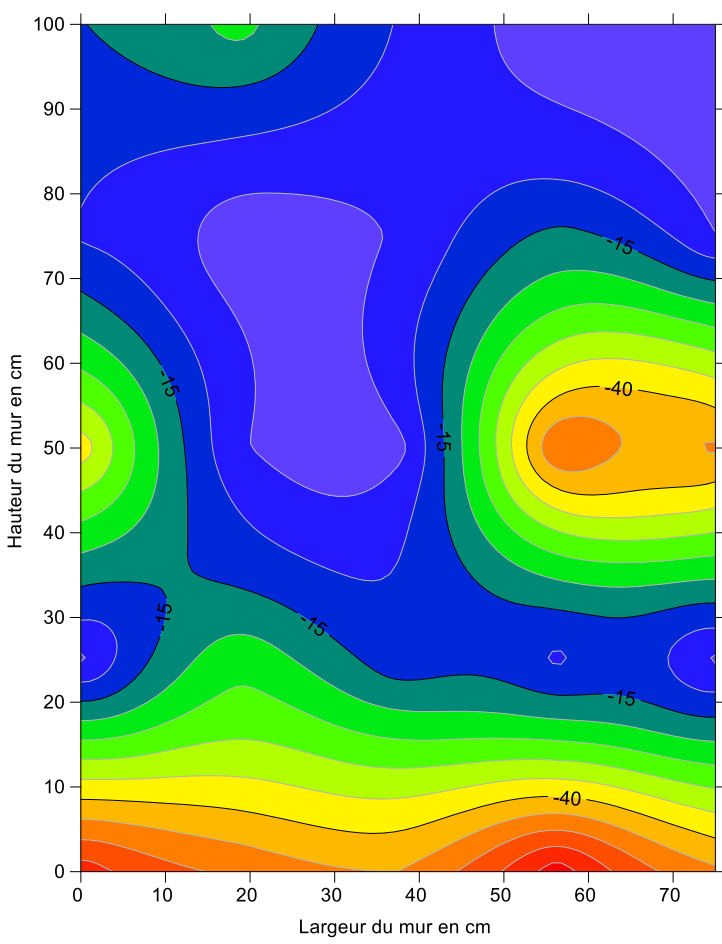

Fig. 19. Cartographie de potentiel OFF après $24 \mathrm{~h}$ de dépolarisation du côté de l'ANODE (mV/ECS).

rapport à la moyenne sont identiques à celles tracées avant les cycles de polarisation et dépolarisation. Ce résultat confirme d'une part l'intérêt de la mesure en relatif qui ne dépend pas des conditions hydriques de l'élément de béton armé mais confirme surtout que la protection cathodique ne conduit pas une uniformisation du champ de potentiel dans le cas de la corrosion initiée par les chlorures. On pourra sans doute argumenter sur le fait que la polarisation n'a duré que 50 jours, mais il est certain qu'on ne peut pas avoir de repassivation des aciers en présence de chlorures et que donc le champ de potentiel restera non uniforme. Ce résultat n'est pas favorable à l'utilisation d'un critère de potentiel car sa vérification sera grandement dépendante de l'endroit où se fait la mesure ${ }^{1}$.

Les cartes de potentiel réalisés du côté de l'anode après $24 \mathrm{~h}$ puis $7 \mathrm{j}$ de dépolarisation sont présentées figures 19 et 20 respectivement. On retrouve des cartes marquées par une forte hétérogénéité comme avant polarisation.

Le suivi de la dépolarisation après la coupure du système de PC (Fig. 21) a été réalisé en utilisant un potentiostat-galvanostat VERSASTAT3 ${ }^{\circledR}$ connecté à l'électrode de manganèse $(\mathrm{Mn})$ noyée dans la paroi. Le potentiel libre recueilli à l'emplacement de l'électrode dans la paroi avant d'appliquer la protection cathodique, appelé $E_{0}$, était $-430 \mathrm{mV} / \mathrm{ECS}$. Comme observé dans la figure 21 , le potentiel $E_{O F F}$ est d'environ $-660 \mathrm{mV} / \mathrm{ECS}$, ce qui signifie que même avec un courant 10 fois plus grand que

\footnotetext{
1 Attention, ce constat pourra être différent dans le cas de la corrosion induite par la carbonatation avec une protection cathodique pouvant conduire à une repassivation des zones initialement corrodée par apport des ions $\mathrm{OH}^{-}$qui pourraient faire remonter le $\mathrm{pH}$ vers des valeurs où l'acier redevient passif.
}

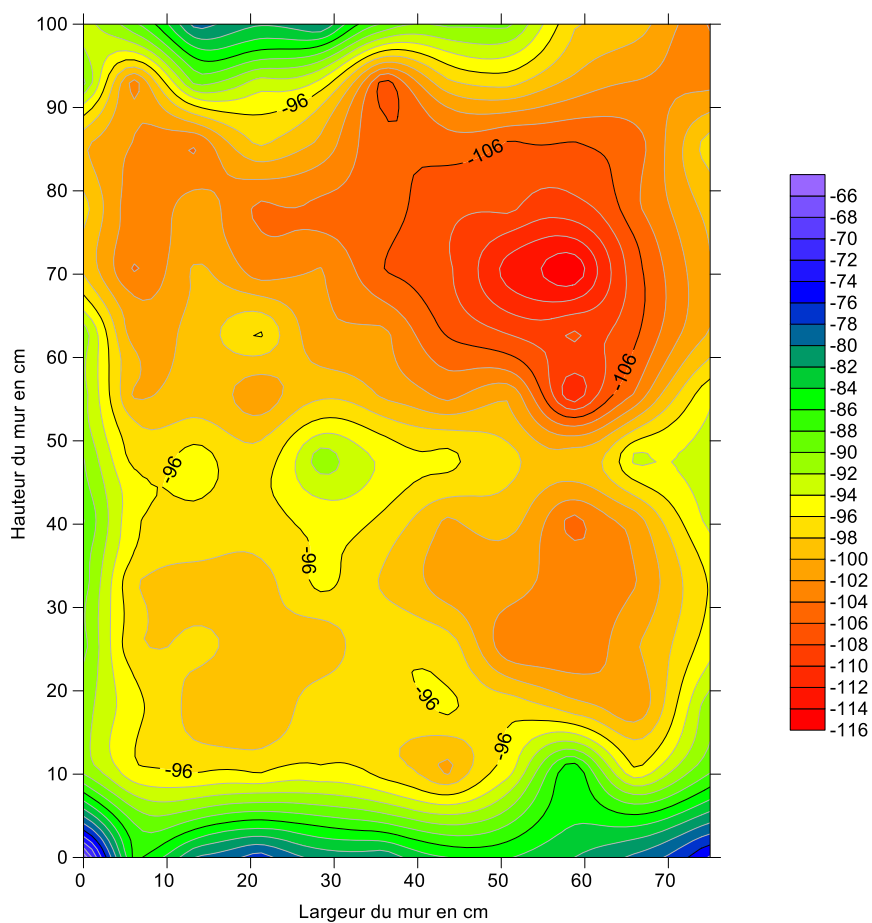

Fig. 20. Cartographie de potentiel OFF après $7 \mathrm{j}$ de dépolarisation du côté de l'ANODE (mV/ECS).

celui qui a stoppé la corrosion (évalué comme un courant nul entre l'armature horizontale 3 et le reste du ferraillage) le premier critère par rapport à la norme EN ISO 12696 n'est pas atteint. Néanmoins, dans ce cas l'emplacement de l'électrode de référence interne joue un rôle important dans ce critère : si l'électrode de manganèse avait été insérée à proximité de la zone de corrosion dans le mur, le premier critère serait atteint. $\mathrm{Au}$ contraire, si l'électrode de manganèse avait été insérée dans un des coins du mur, le premier critère serait tout à fait impossible à réaliser, même sous une polarisation encore plus élevée. Par ailleurs, si on cherche à vérifier le premier critère dans un des coins du mur alors, compte-tenu du gradient de potentiel de $200 \mathrm{mV}$ visualisé dans la figure 15 , on obtiendrait des valeurs plus négatives que le critère de fonctionnement des structures précontraintes (pas de potentiel plus faible que $-900 \mathrm{mV} / \mathrm{Ag} / \mathrm{AgCl} / \mathrm{KCl} 0,5 \mathrm{M})$.

Cependant, le deuxième critère est clairement vérifié pour $4 \mathrm{~h}$ et $24 \mathrm{~h}$ a fortiori: par ailleurs, la dépolarisation est supérieure à $300 \mathrm{mV}$. Ainsi, la grande différence entre la valeur prescrite par la norme $(100 \mathrm{mV})$ et celle enregistrée $(300 \mathrm{mV})$ est discutable : est-ce le résultat d'une polarisation excessive?

On peut remarquer aussi que, très peu de temps après la coupure du courant de protection, le potentiel mesuré $(-288 \mathrm{mV} / \mathrm{ECS})$ est supérieur à la valeur initiale libre $E_{0}$. De plus, ce potentiel reste plus élevé $(-268 \mathrm{mV} / \mathrm{ECS})$ même 20 jours après la coupure du système de PCCI.

$\mathrm{Au}$ final bien qu'on ait clairement appliqué une polarisation bien plus importante que nécessaire, le gradient de potentiel sur l'ensemble du réseau d'armature semble rester le même et identique au potentiel naturel (Fig. 10), lors de la polarisation (Fig. 14) et de la postdépolarisation (Fig. 18). 


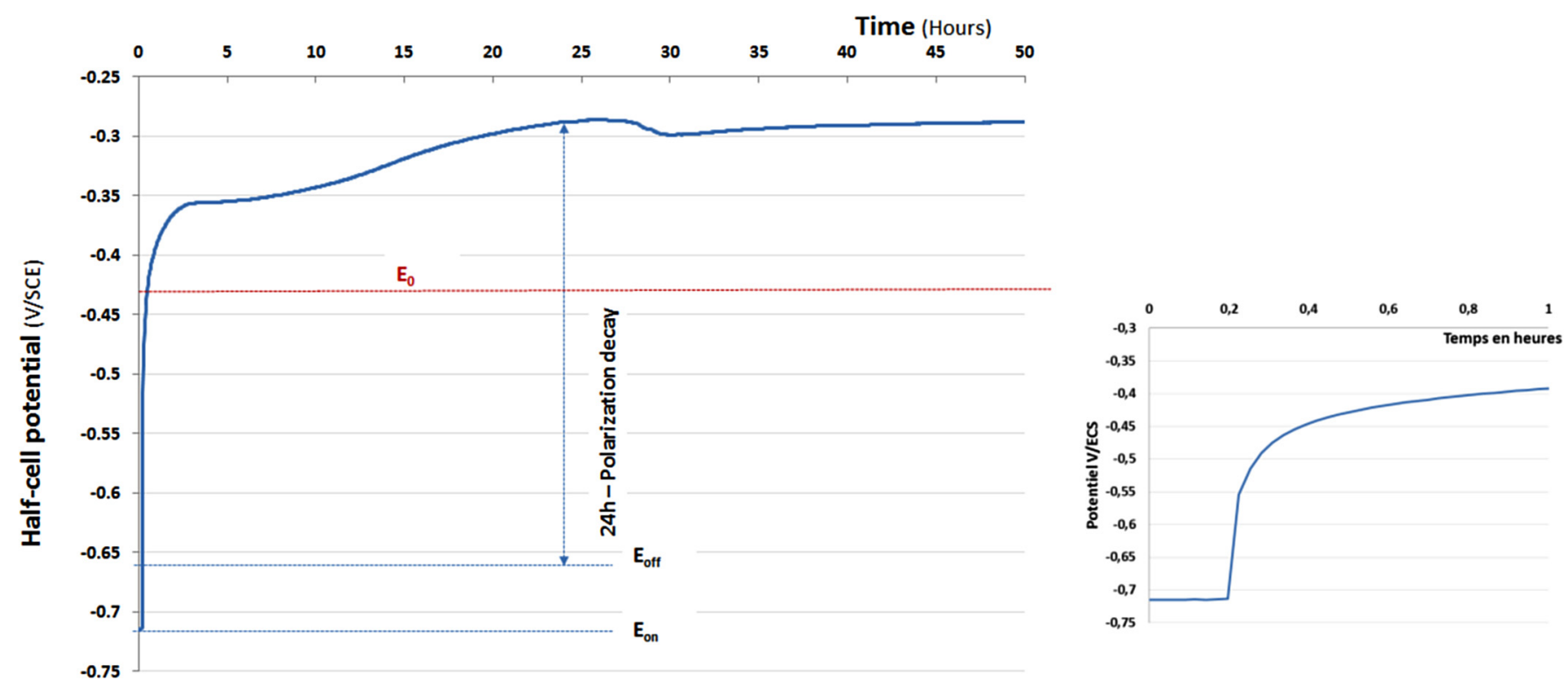

Fig. 21. Courbe de dépolarisation du mur M3PC mesurée à l'aide de l'électrode interne au manganèse sur une durée de $50 \mathrm{~h}$ avec un zoom sur la première heure

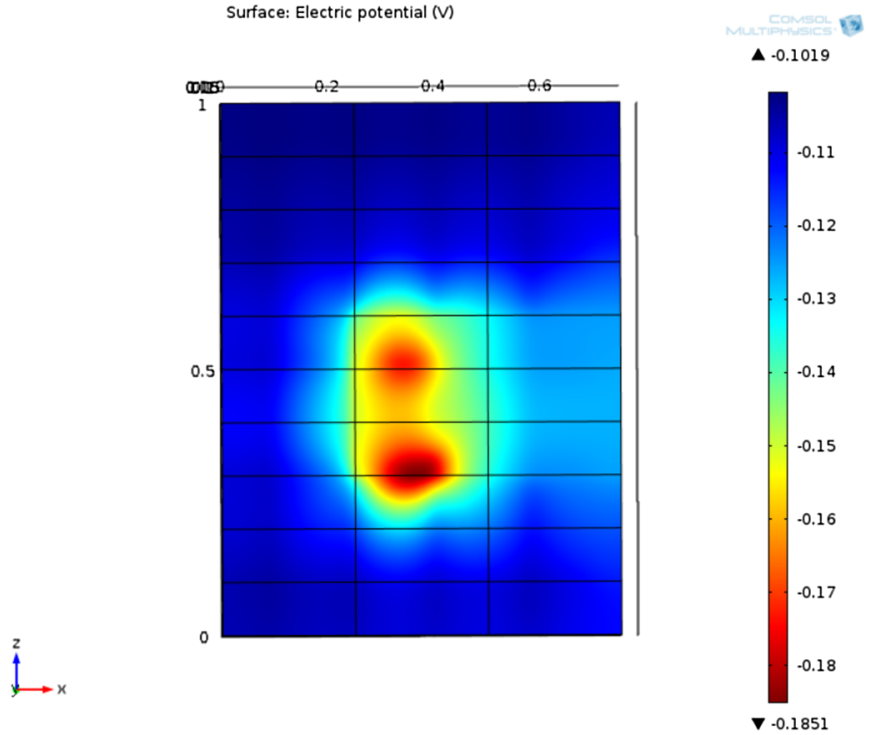

Fig. 22. Carte de potentiel en surface simulée pour le système de corrosion non uniforme libre.

\section{Résultats de l'étude numérique}

Afin d'effectuer les simulations les plus réalistes possibles et pour tenir compte de l'environnement sec du mur (salle de TP - même si on humidifie de temps en temps les surfaces du mur), une résistivité électrique de $500 \mathrm{ohm} . \mathrm{m}$ a été utilisée sauf pour la partie contaminée par les chlorures autour du point de corrosion central, où on a utilisé 100 ohm.m. Malgré toutes ces précautions, Il doit être noté que les valeurs numériques simulées restent empreintes de quelques incertitudes.

La première simulation traite du système de macropiles correspondant à la corrosion libre (ou naturelle). La

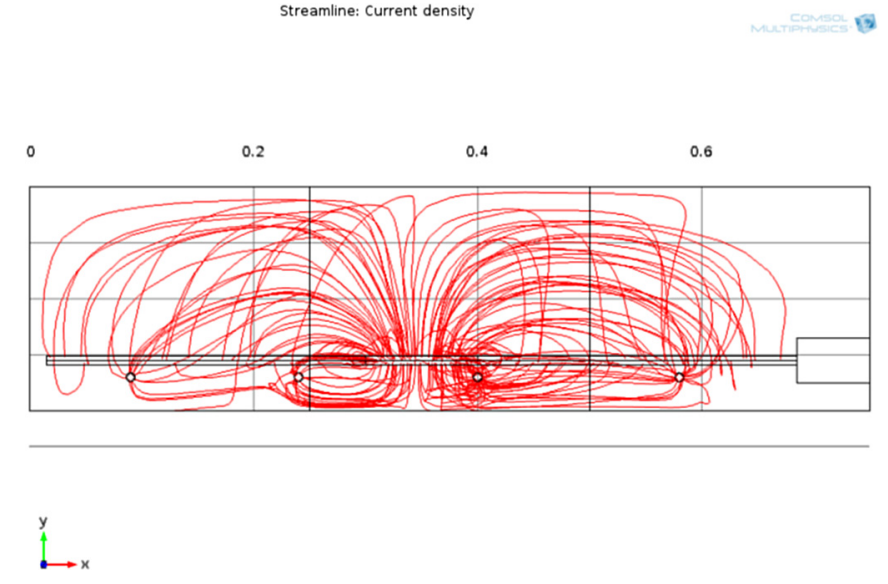

Fig. 23. Carte de courant de corrosion simulée pour le système de corrosion non uniforme libre.

corrosion a été simulée principalement sur les barres horizontales 3 et 4 et un peu sur la barre verticale 3 (Fig. 8) afin de reproduire qualitativement le motif de potentiel expérimental présenté (Fig. 9).

La carte libre de potentiel simulé recueillie sur la face avant de la paroi est représentée dans la figure 22 .

La carte des courants de corrosion est présentée figure 23. Le calcul du courant de corrosion issu de la barre horizontale 3 donne une valeur de 26 microA et lorsque qu'on calcule le courant total des zones de corrosion simulées, on obtient 54 microA. Ces valeurs sont à rapprocher des mesures expérimentales qui donnent 54 microA pour la barre 3 seule et 69 microA pour les deux barres horizontales. On dira que pour une première approche, le modèle numérique donne des résultats cohérents avec l'expérience, même si certains paramètres ne sont pas connus comme par exemple la dimension des zones actives. 


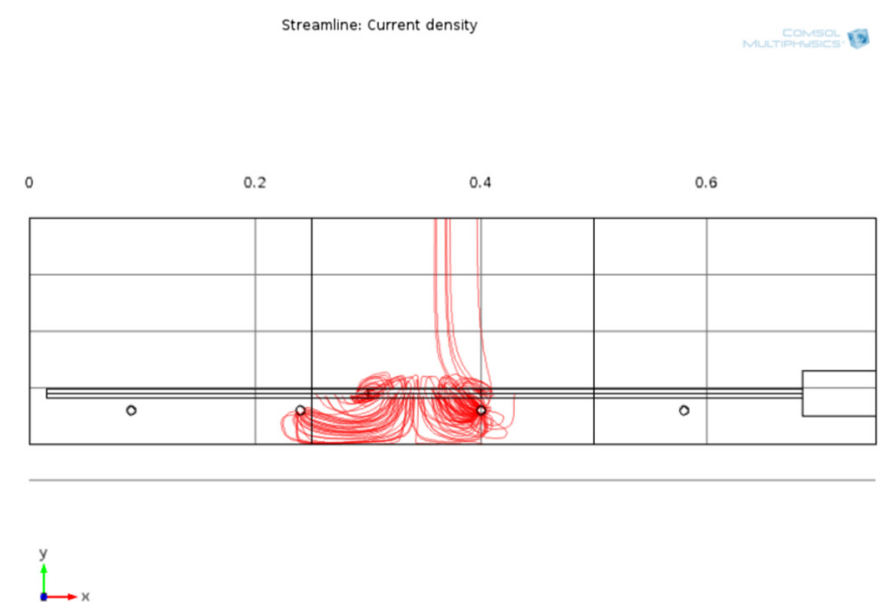

Fig. 24. Courant de corrosion modélisé pour une polarisation correspondant à un courant de $0,3 \mathrm{~mA}$.

Streamline: Current density

0.2 0.4

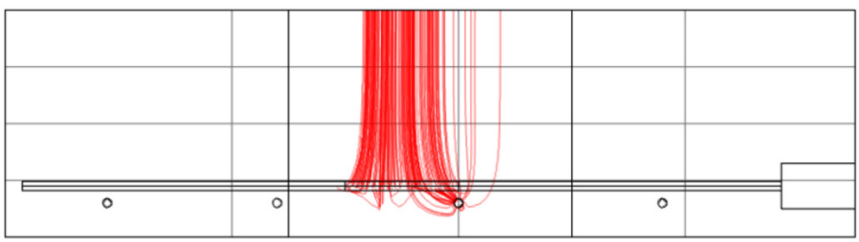

$\stackrel{x}{\longrightarrow} \rightarrow$

Fig. 25. Courant de corrosion modélisé pour une polarisation correspondant à un courant de $0,7 \mathrm{~mA}$ : la corrosion est stoppée.

Dans un second temps, le modèle numérique a été modifié afin d'appliquer la protection cathodique par l'intermédiaire du treillis en titane située sur le côté opposé de la paroi. La perturbation électrochimique a simplement été simulée en mettant en œuvre un potentiel flottant du côté de l'anode en titane, avec les contraintes suivantes:

- premièrement trouver le courant de protection qui annule le courant de corrosion sortant de la barre 3 pour le comparer avec la valeur expérimentale;

- deuxièmement, avoir un courant de protection appliquée identique à la seconde phase expérimentale qui était de $3 \mathrm{~mA}$ (équivalent à $15 \mathrm{~mA} / \mathrm{m}^{2}$ de surface d'armatures).

Les figures 24 et 25 montrent le champ de courant de corrosion pour des intensités de courant imposé de $0,3 \mathrm{~mA}$ et $0,7 \mathrm{~mA}$ respectivement. Numériquement, il faut atteindre une intensité de $0,7 \mathrm{~mA}$ pour que le courant de corrosion s'annule, ce qui est supérieur à la valeur expérimentale $(0,3 \mathrm{~mA})$ mais cela reste du même ordre de grandeur.

Pour un courant de protection de $0,3 \mathrm{~mA}, 0,7 \mathrm{~mA}$ puis $3 \mathrm{~mA}$, le tracé de la carte de potentiel «ON » est présenté dans les figures $26-28$ respectivement.

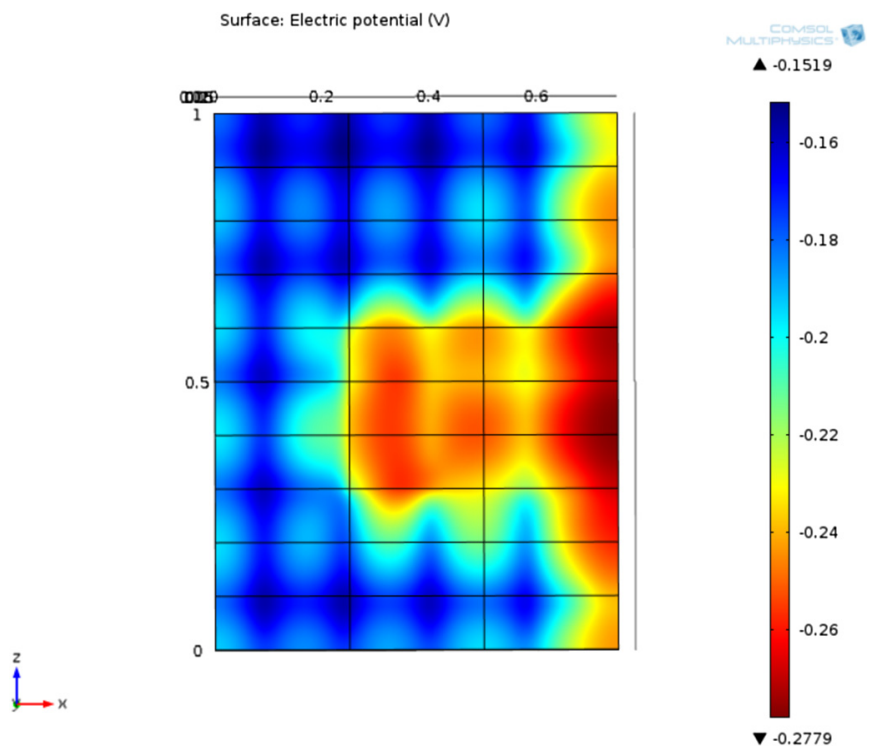

Fig. 26. Carte de potentiel «ON» pour un courant de $0,3 \mathrm{~mA}$ (densité de $1,5 \mathrm{~mA} / \mathrm{m}^{2}$ ).

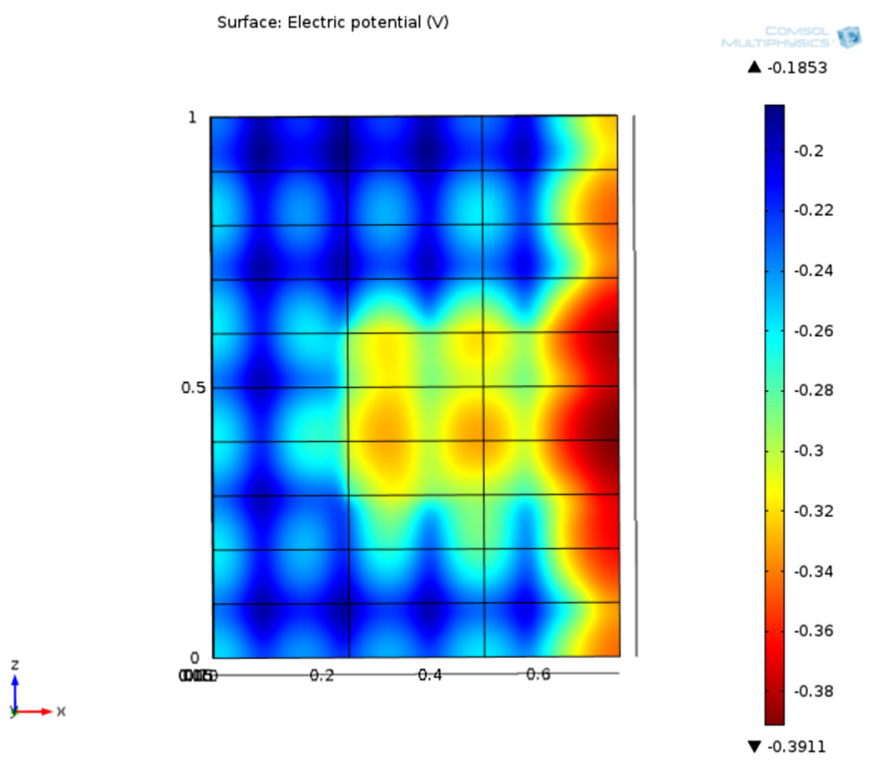

Fig. 27. Carte de potentiel « $\mathrm{ON} »$ pour un courant de $0,7 \mathrm{~mA}$ (densité de $4,5 \mathrm{~mA} / \mathrm{m}^{2}$ ).

La simulation numérique est qualitativement plus proche de la carte de potentiel «ON » expérimentale (Fig. 11) pour un faible courant de protection (Fig. 26) avec un champ de potentiel qui devient plus négatif dans la partie droite du mur. Lorsque le courant de protection augmente, c'est surtout le fantôme du réseau d'armature qui devient plus visible et la partie droite du mur montre des potentiels très négatifs que l'on constate aussi expérimentalement.

Ce travail de simulation utilisant les lois physiques de base qui régissent les phénomènes électrochimiques confirme que la polarisation des systèmes de corrosion de type macro-piles est un vrai problème physique $3 \mathrm{D}$ et ne peut pas être approchée par des modèles 1D. Les 


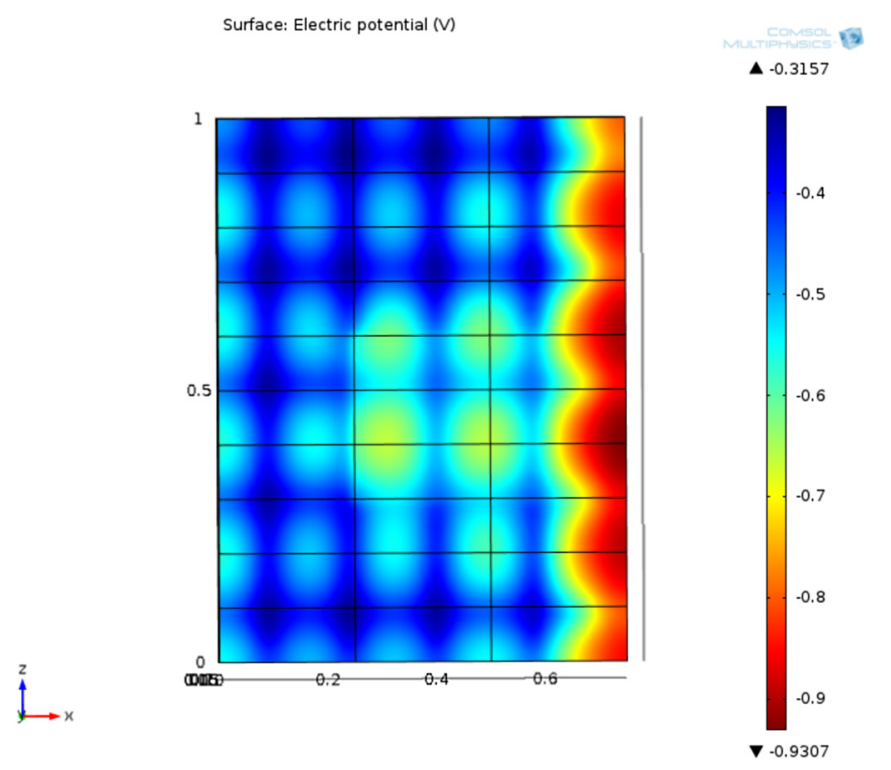

Fig. 28. Carte de potentiel «ON» pour un courant de $3 \mathrm{~mA}$ (densité de $15 \mathrm{~mA} / \mathrm{m}^{2}$ ).

simulations numériques confirment également que l'uniformité du champ de potentiel à l'interface acier-béton n'est physiquement pas réalisable en appliquant un courant de protection de la surface du béton. Ce point a déjà été mis en évidence par Saguës et Kranc [31]. Le principe général de la protection cathodique n'a pas à être discuté, mais une attention particulière doit être accordée à la réponse non uniforme des systèmes de macro-piles car il remet en question, entre autres, le choix de la position des témoins où la performance du système de $\mathrm{PC}$ est évaluée et la valeur $E_{O F F}$ utilisée pour cette évolution. En effet, $E_{O F F}$ résulte d'une vision $1 \mathrm{D}$ de la réponse de l'interface acier-béton à une coupure de courant et ne prend pas en compte la phénoménologie de la corrosion par macro-piles [31].

\section{Conclusions-perspectives}

Sans remettre en cause l'efficacité de la protection cathodique, les résultats sur les études expérimentale et numérique, présentées dans cet article, font apparaître qu'une amélioration est possible au sujet des critères d'efficacité d'une protection cathodique en développant une approche théorique et numérique plus importante.

Le critère d'efficacité exprimé en potentiel «instant $\mathrm{OFF} \gg(-720 \mathrm{mV} / \mathrm{Ag} / \mathrm{AgCl} 0,5 \mathrm{M})$ ne semble pas avoir de signification particulière pour un système de corrosion par macro-piles en raison, en particulier, de la non-uniformité du champ de potentiel de corrosion libre, du champ de potentiel sous protection cathodique, et du champ de potentiel pendant la dépolarisation. Dans le cas d'un tel système de corrosion par macro-piles, le ratio entre les surfaces anodiques et cathodiques et la résistivité du béton vont jouer le rôle le plus important sur le processus de corrosion. Le courant de protection à appliquer dépend donc du gradient de potentiel dans le système et ne peut donc pas être relié à une mesure ponctuelle du potentiel.
L'étude expérimentale réalisée sur le mur M3PC et présentée dans cet article a montré que la protection cathodique pouvait être réalisée alors que le potentiel 《instant $\mathrm{OFF}$ » de l'acier était voisin de -250 à $-300 \mathrm{mV} /$ $\mathrm{Ag} / \mathrm{AgCl}$ 0,5 M. Par ailleurs, le champ de potentiel avant, pendant et après polarisation cathodique est non uniforme. Une étude complémentaire réalisée par Laurens et François [32] mesurant simultanément les potentiels $E_{O F F}$ en six points différents du mur M3PC a confirmé la nonuniformité des potentiels $E_{O F F}$.

Le premier critère correspond à une restauration des conditions de passivité. Bertolini et al. [33] avaient déjà démontré qu'il n'est pas nécessaire de restaurer les conditions de passivité pour réduire la corrosion par protection cathodique, en abaissant le potentiel sous la valeur donnée par la loi de Nernst $(-720 \mathrm{mV} / \mathrm{Ag} / \mathrm{AgCl} /$ $0,5 \mathrm{M})$. Mais les résultats présentés dans cet article vont audelà en indiquant que la vérification du premier critère de protection cathodique définit par la norme EN ISO 12696 n'est pas pertinente, parce qu'elle est basée implicitement sur l'équipotentialité du champ de potentiel sous protection cathodique.

Les critères de dépolarisation à $24 \mathrm{~h}$ ou plus sont aussi concerné par l'existence d'un gradient de potentiel avant et après dépolarisation. Pour les exemples traités dans cet article: il apparaît que la protection cathodique semblait effective sans que les critères de dépolarisation soient vérifiés ou alors que l'application des densités de courants préconisées par la norme EN ISO 12696 conduit à des dépolarisations au moins 3 fois supérieures aux critères, ce qui voudrait dire que ces critères sont trop conservatifs. Ce point, cependant, mériterait une vérification à l'échelle de la structure. En revanche, les critères de dépolarisation ne prennent pas en compte le fait que le champ de polarisation est non uniforme et que donc la modification des courants de corrosion pendant l'application de la protection cathodique ne peut pas être seulement appréciée par une mesure en courant coupé. La vérification des second et troisième critères de dépolarisation dépend aussi de la position des électrodes ou sondes de contrôles. En conséquence, la question de la mesure de la vérification de l'efficacité de la protection cathodique est ouverte : estce que les critères de dépolarisation vérifiés en un point signifient qu'ils sont vérifiés en tout point? et est-ce que la vérification des critères en un point garanti qu'il n'y a pas une sur-polarisation en un autre point?

Le critère de bon fonctionnement exprimé en potentiel «instant $\mathrm{OFF}$ » $(-900 \mathrm{mV} / \mathrm{Ag} / \mathrm{AgCl} 0,5 \mathrm{M})$ pour s'assurer de la non-production d'hydrogène à la cathode n'a pas non plus de signification pour un système de corrosion par macro-piles en raison en particulier de la non-uniformité du champ de potentiel de corrosion libre, du champ de potentiel sous protection cathodique, et du champ de potentiel pendant la dépolarisation. Ce n'est pas parce que ce critère sera vérifié en un point qu'on pourra garantir qu'une zone de la structure n'est pas en deçà de cette valeur.

En perspective de ce travail, on peut proposer quelques pistes d'amélioration du contrôle de l'efficacité de la protection cathodique, concernant les critères de performance et le développement des outils numériques. 
Il faudra sans doute envisager de ne pas définir de critères de protection universels et au contraire d'adapter ces critères à la géométrie et l'environnement du système de corrosion localisée différent pour chaque structure, en développant une modélisation 3D de leur comportement. Ce point doit être le moteur des formations et certification de niveau 3 et niveau 4 par rapport à la norme EN ISO 15257:2017.

Le challenge le plus important pour les recherches futures serait d'être capable de faire une analyse inverse du fonctionnement d'un système de corrosion par macro-piles et d'un système de protection cathodique. En effet, on peut supposer que la cartographie de potentiel réalisée en surface sur un ouvrage résulte d'une configuration unique de corrosion: répartition et surfaces des zones anodiques et cathodiques. Ainsi une analyse inverse utilisant la méthode des éléments finis permettrait d'identifier précisément l'état de corrosion.

De même, la cartographie de potentiel ON pendant la protection cathodique devrait, également par analyse inverse, permettre de savoir si la corrosion est effectivement «stoppée» ou suffisamment réduite pour être négligeable par rapport à la durée de vie de l'ouvrage.

On peut se poser la question de savoir si une cartographie de potentiel correspond à une solution unique de l'état de corrosion dans la structure car finalement ce n'est qu'une vision partielle des effets de la corrosion : dans ce cas la solution pourrait être de réaliser une autre cartographie en ne prenant pas comme référence le réseau d'armatures mais une autre référence comme par exemple une électrode qui serait insérée par forage dans la structure. On aurait ainsi une seconde cartographie et il devient probable qu'il existerait une solution unique de l'état de corrosion conduisant à ces deux cartographies.

\section{References}

1. R. François, J.C. Maso, Effect of damage in reinforced concrete on carbonation or chloride penetration, Cem. Concr. Res. 18, 961 (1988)

2. U. Angst, R.D. Hooton, J. Marchand, C.L. Page, R.J. Flatt, B. Elsener, C. Gehlen, J. Gulikers, Present and future durability challenges for reinforced concrete structures, Mater. Corros. 63, 1047 (2012)

3. R. Polder, W.H.A. Peelen, W. Courage, Non-traditional assessment and maintenance methods for aging concrete structures - technical and non-technical issues, Mater. Corros. 63, 1147 (2012)

4. U. Angst, B. Elsener, C.K. Larsen, Ø. Vennesland, Critical chloride content in reinforced concrete $-\mathrm{A}$ review, Cem. Concr. Res. 39, 1122 (2009)

5. T.A. Söylev, François R., Corrosion of reinforcement in relation to presence of defects at the interface between steel and concrete, J. Mater. Civ. Eng. 17, 447 (2005)

6. L. Yu, R. François, V.H. Dang, V. L'Hostis, R. Gagné, Distribution of corrosion and pitting factor of steel in corroded RC beams, Constr. Build. Mater. 95, 384 (2015)

7. A.T. Horne, I.G. Richardson, R.M.D. Brydson, Quantitative analysis of the microstructure of interfaces in steel reinforced concrete, Cem. Concr. Res. 37, 1613 (2007)
8. A. Kenny, A. Katz, Statistical relationship between mix properties and the interfacial transition zone around embedded rebar, Cem. Concr. Compos. 60, 82 (2015)

9. T.A. Söylev, R. François, Quality of steel-concrete interface and corrosion of reinforcing steel, Cem. Concr. Res. 33, 1407 (2003)

10. R. François, S. Laurens, F. Deby, La corrosion et ses conséquences dans les ouvrages en béton armé, ISTE Press - Elsevier, 2018, ISBN: 9781785482342

11. U. Angst, M. Büchler, On the applicability of the SternGeary relationship to determine instantaneous corrosion rates in macro-cell corrosion, Mater. Corrosion 66, 1017 (2015)

12. S. Laurens, P. Hénocq, N. Rouleau, F. Deby, E. Samson, J. Marchand, B. Bissonnette, Steady-state polarization response of chloride-induced macrocell, corrosion systems in steel reinforced concrete-numerical and experimental investigations, Cem. Concr. Res. 79, 272 (2016)

13. H. Davy, On the corrosion of copper sheeting by seawater, and on methods of preventing this effect, and on their application to ships of war and other ships, Proc. R. Soc. 114, 151 ( 1824)

14. R.B. Polder, Cathodic protection of reinforced concrete structures in The Netherlands-experience and developments, HERON, 43(1), 3 (1998)

15. P. Pedeferri, Cathodic protection and cathodic prevention, Constr. Build. Mater. 10, 391 (1995)

16. L. Bertolini, A tribute to Pietro Pedeferri's contribution to the knowledge on corrosion of steel in concrete and its prevention. Mater. Corros. 62(2), 96 (2011)

17. L. Bertolini, F. Bolzoni, A. Cigada, T. Pastore, P. Pedeferri, Cathodic protection of new and old reinforced concrete structures. Corros. Sci. 35(5-8), 1633 (1993)

18. L. Bertolini, B. Elsener, P. Pedeferri, E. Redaelli, R. Polder, Corrosion of steel in concrete: Prevention, diagnosis, repair (2nd ed.), Wiley VCH, Weinheim, Germany, 2013

19. Norme EN ISO 12696:2016, Cathodic protection of steel in concrete ISO 12696 2012, ISO, Vienna, 2016, 45 p

20. M. Pourbaix, Atlas of electrochemical equilibria in aquaous solutions, NACE, Houston, TX, 1974

21. Han M.K. et al., Cathodic Protection of Concrete Bridge Components, Strategic Highway Research Program, Progress Report for Contract SHRP-87-C-102B, 1989

22. NACE 1990 Standard Recommended Practice, NACE Standard RP0290-90, Item No. 53072, April, 1990

23. J.E. Bennett, T.A. Mitchell, Depolarization of Cathodically Protected Reinforcing Steel in Concrete, Paper No.373, NACE Corrosion/89, New Orleans, 1989

24. M. Funishashi, J.B. Bushman, Technical review of $100 \mathrm{mV}$ polarization shift criterion for reinforcing steel in concrete, Corrosion 47(5), (1989)

25. R.C.G. Laird, Performance evaluation testing of conductive coating cathodic protection systems on thin parking garage slabs, Paper No. 553, NACE Corrosion/91, Cincinnati, 1991

26. NACE International, CP-2 Cathodic protection technician course manual, Nace, Houston, 2000

27. U. Angst, M. Büchler, B. Martin, H.G. Schöneich, G. Haynes, S. Leeds, F. Kajiyama, Cathodic protection of soil buried steel pipelines - a critical discussion of protection criteria and threshold values, Mater. Corros. 67(11), 1135 (2016) 
28. S. Chakri, P. David, I. Frateur, A. Galtayries, P. Marcus, E. Sutter, B. Tribollet, V. Vivier, S. Zanna, Effet de la composition chimique de la solution interstitielle de bétons jeunes sur la passivation d'un acier doux, Mattech 103, 209 (2015), EDP Sciences, 2015, DOI: 10.1051/mattech/ 2015016, www.mattech-journal.org

29. R. François, G. Arliguie, D. Bardy, Electrode potential measurements of concrete reinforcement for corrosion evaluation, Cem. Concr. Res. 24(3), 401 (1994)

30. R. François, G. Arliguie, Z. Hamdan, Utilisation de l'interprétation en valeur relative des mesures de potentiel d'électrode des armatures du béton, Ann. I.T.B.T.P. 488, 1 (1990)
31. A.A. Sagües, S.C. Kranc, On the determination of polarization diagrams of reinforcing steel in concrete, Corrosion 48, 624 (1992)

32. S. Laurens, R. François, Cathodic protection in reinforced concrete structures affected by macrocell corrosion: a discussion about the significance of the protection criteria, RILEM Tech. Lett. 2, (2017), https://doi.org/10.21809/ rilemtechlett.2017.38

33. L. Bertolini, F. Bolzoni, P. Pedeferri, L. Lazzari, T. Pastore, Cathodic protection and cathodic prevention in concrete: principles and applications, J. Appl. Electrochem. 28, 1321 (1998)

Citation de l'article : Raoul François, Protection cathodique appliquée au béton armé aérien: étude expérimentale et modélisation, Matériaux \& Techniques 106, 201 (2018) 